\title{
Width of the Homoclinic Zone in the Parameter Space for Quadratic Maps
}

\author{
V. Gelfreich and V. Naudot
}

\section{CONTENTS}

1. Introduction

2. Main Results

3. Computing the Width of the Homoclinic Zone

4. Validation of the Numerical Method

Acknowledgments

References

2000 AMS Subject Classification: Primary 37D45, 37E30, 37G10

Keywords: Bogdanov map, Bogdanov-Takens bifurcation, Hénon map, separatic splitting, splitting function
We study several families of planar quadratic diffeomorphisms near a Bogdanov-Takens bifurcation. For each family, the skeleton of the associated bifurcation diagram can be deduced from the interpolating flow. However, a zone of chaos confined between two lines of homoclinic bifurcation that are exponentially close to one another is observed. The goal of this paper is to test numerically an accurate asymptotic expansion for the width of this chaotic zone for different families.

\section{INTRODUCTION}

In this paper we study homoclinic bifurcations in the unfolding of a diffeomorphism near a fixed point of Bogdanov-Takens type. To begin with, we consider a planar diffeomorphism $F: \mathbb{R}^{2} \rightarrow \mathbb{R}^{2}$ with the origin as a fixed point and where

$$
d F(0,0)=\mathbb{I} \mathrm{d}+\mathrm{N}
$$

where $\mathrm{N} \not \equiv 0$ is nilpotent. The origin is said to be a fixed point of Bogdanov-Takens type. This latter terminology is better known for a singularity of a vector field $X$ with linear part having double zero eigenvalues and a nonvanishing nilpotent part. Since this singularity is of codimension 2, i.e., is twice degenerate, a generic unfolding will depend on two parameters, say $(\mu, \nu)$.

In the case of a vector field, such unfolding has been studied in [Arnol'd 83, Takens 74], and for maps it has been studied in [Broer et al. 96, Broer et al. 92]. For completeness, the corresponding bifurcation diagram is revisited in Figure 1 (left): a curve of homoclinic bifurcation emanates from the origin, below a curve of Hopf bifurcation; see [Broer et al. 91] for the terminology and more details.

For parameters located between these two curves, the corresponding dynamics possess a stable limit cycle. Finally, for the parameter $\{\mu=0\}$ on the ordinate, a saddle node occurs; see also [Broer et al. 91] for more details.

(c) A K Peters, Ltd. $1058-6458 / 2009 \$ 0.50$ per page Experimental Mathematics 18:4, page 409 

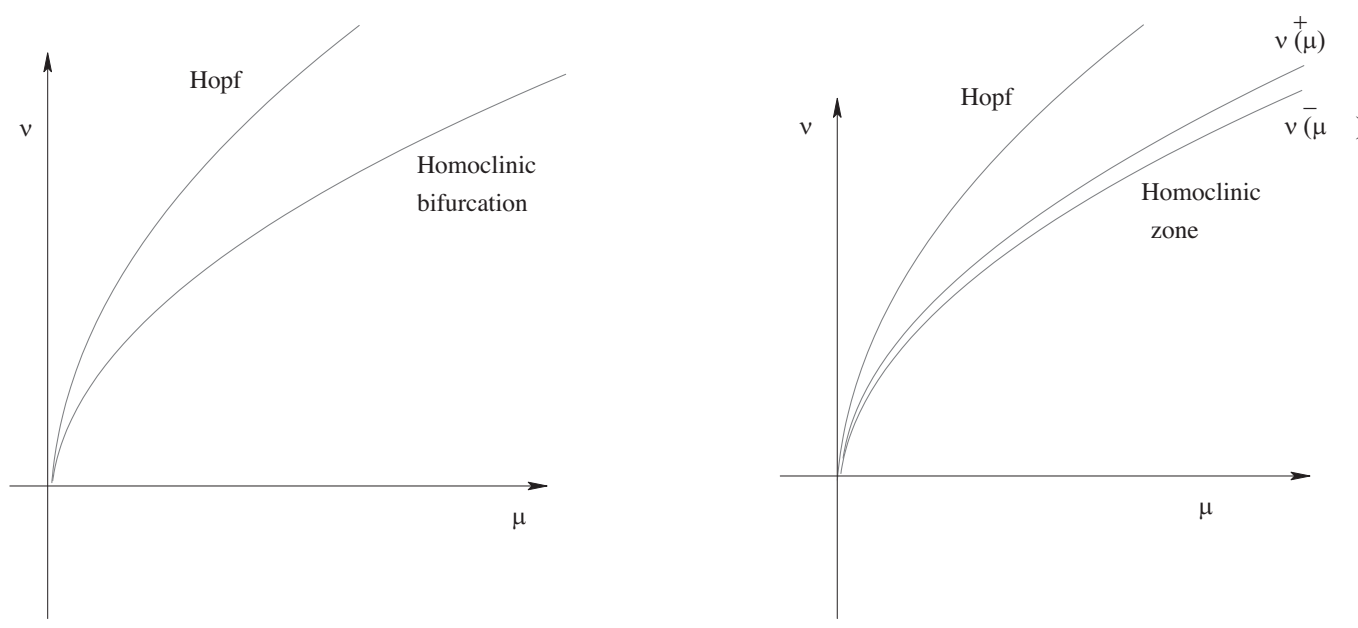

FIGURE 1. The Bogdanov-Takens bifurcation for a flow (left) and for a diffeomorphism (right).

The Bogdanov-Takens bifurcation plays an important role in dynamical systems, for instance from the bifurcation-theoretic point of view. Given any dynamical system depending on a parameter, the structure of the bifurcation set can often be understood by the presence of several high-codimension points that act as organizing centers. Starting from the presence of (degenerate or nondegenerate) Bogdanov-Takens points, we can initiate the search for subordinate bifurcation sets such as Hopf bifurcation sets or homoclinic bifurcation sets. In this paper, we consider a nondegenerate Bogdanov-Takens point.

For the map $F$, an unfolding theory is developed in [Broer et al. 96, Broer et al. 92]. It is very similar to the case of a flow. To be more precise, any unfolding

$$
F_{\mu, \nu}: \mathbb{R}^{2} \rightarrow \mathbb{R}^{2}, \quad(x, y) \mapsto\left(x_{1}, y_{1}\right),
$$

of the map $F$ (where $(\mu, \nu) \in \mathbb{R}^{2}$ and $F=F_{0,0}$ ) can be embedded in a nonautonomous and periodic family of vector fields $X_{\mu, \nu}$. The diffeomorphism coincides with the time-1 map of that vector field; see also [Takens 74].

Using an averaging theorem [Neishtadt 84], the dependence on time is removed to exponentially small terms. Moreover, one can show that $F_{\mu, \nu}$ is formally interpolated by an autonomous vector field $\tilde{X}_{\mu, \nu}$; see [Gelfreich 03]. This latter can be used to study the bifurcations of fixed points of $F_{\mu, \nu}$. Both approaches move the difference between these two types of bifurcations beyond all algebraic order.

Although all the Taylor coefficients of $\tilde{X}_{\mu, \nu}$ can be written, there is no reason to expect convergence of the corresponding series, since the dynamics for a planar diffeomorphism can be much richer than the dynamics of a planar vector field. In the real analytic theory, this difference is exponentially small [Broer and Roussarie 01, Gelfreich 03].

As stated above, for diffeomorphisms, the bifurcation diagram (Figure 1, right) is essentially the same. However, there is no reason to expect a single homoclinic curve, since a homoclinic orbit may be transverse and therefore persists. We observe a separatrices splitting, and instead of a single homoclinic curve, one observes two curves $\nu^{+}(\mu)$ and $\nu^{-}(\mu)$ respectively corresponding to the first and last homoclinic tangencies. If a parameter $(\mu, \nu)$ is (strictly) located in the region between those two curves, then the map $F_{\mu, \nu}$ possesses transverse homoclinic trajectories. On the lower and upper boundaries, the homoclinic connection becomes nontransverse. Understanding the width of this region is the main goal of this paper.

Before going any further, we set the following preliminaries. Without loss of generality and up to an analytic change of coordinates, one has

$$
x_{1}=x+y, \quad y_{1}=y+f_{\mu, \nu}(x, y),
$$

where

$$
f_{0,0}(0)=0=\frac{\partial f_{0,0}}{\partial x}(0,0)=\frac{\partial f_{0,0}}{\partial y}(0,0) .
$$

We shall assume that

$$
\frac{\partial^{2} f_{0,0}}{\partial x^{2}}(0,0) \neq 0 .
$$

By the implicit function theorem, there exists $\tilde{x}(\mu, \nu)$ such that

$$
\frac{\partial f_{\mu, \nu}}{\partial x}(\tilde{x}(\mu, \nu), 0) \equiv 0 .
$$


Applying a conjugacy of the form $x=\bar{x}+\tilde{x}_{\mu, \nu}, y=\bar{y}$ (and after removing the bars) amounts to writing

$$
\begin{aligned}
f_{\mu, \nu}(x, y)= & -b_{00}(\mu, \nu)+b_{20}(\mu, \nu) x^{2}+b_{01}(\mu, \nu) y \\
& +b_{11}(\mu, \nu) x y+\text { h.o.t. }(x, y)
\end{aligned}
$$

h.o.t. $(x, y)$ stands for the higher-order terms in $x$ and $y$. From $(1-2)$, we have $b_{20}(0,0) \neq 0$.

By a linear rescaling in the variables $(x, y)$, we can fix $b_{20}(\mu, \nu) \equiv 1$. Furthermore, we put $b_{11}(0,0)=\gamma$ and assume that the map

$$
\begin{aligned}
(\mu, \nu) & \mapsto\left(-f_{\mu, \nu}(0,0),\right. \\
\left.\frac{\partial f_{\mu, \nu}}{\partial y}(0,0)\right) & =\left(-b_{00}(\mu, \nu), b_{01}(\mu, \nu)\right),
\end{aligned}
$$

is a local diffeomorphism near $(0,0)$. From now on, we shall consider $\left(b_{00}, b_{01}\right)$ as our parameters and rename them (again) by $(\mu, \nu)$, i.e., we shall write $\left(b_{00}, b_{01}\right)=$ $(\mu, \nu)$. Therefore, our study concerns the map

$$
f_{\mu, \nu}(x, y)=-\mu+x^{2}+\nu y+\gamma x y+\text { h.o.t. }(x, y),
$$

and we restrict our study to the case in which $\mu>0$.

In [Broer et al. 96] it was shown that

$$
\nu^{ \pm}(\mu)=\frac{5}{7}(\gamma-2) \sqrt{\mu}+\mathcal{O}\left(\mu^{\frac{3}{4}}\right)
$$

In [Gelfreich 03] the following formula is proposed:

$$
\nu^{+}(\mu)-\nu^{-}(\mu)=K(\mu, \gamma-2)\left(\Theta_{\gamma}+\mathcal{O}\left(\mu^{1 / 4} \log \mu\right)\right),
$$

where

$$
K(\mu, \tilde{\gamma})=\frac{5}{6 \sqrt{2} \mu^{\frac{5}{4}}} \cdot e^{-\sqrt{2} \pi^{2} / \sqrt[4]{\mu}} e^{-6 \pi^{2} \tilde{\gamma} / 7}
$$

is referred to as the leading part of the width and $\boldsymbol{\Theta}_{\gamma}$ is an analytic invariant of the map $F_{0,0}$ called a splitting constant; see [Gelfreich and Naudot 06].

The goal of this paper is to establish, numerically, a more accurate formula for the width of the homoclinic zone $\nu^{+}(\mu)-\nu^{-}(\mu)$. The existence of asymptotic expansions for the width of the homoclinic zone is currently an open question. Furthermore, if it does exist, it is very hard to compute analytically. The difficulty here comes from the fact that the normal form of the map coincides with that of the time- 1 map of a vector field. Therefore the difference between the flow and the map is pushed beyond any algebraic order.

In the nearly integrable context, a polynomial asymptotic expansion for the splitting of the separatrices is proposed in [Ramirez-Ros 05]: the author considers the perturbation of a Hamiltonian (elliptic) billiard. The system depends on a perturbation parameter $\varepsilon \geq 0$ and a hyperbolicity parameter $h>0$ and admits four separatrices, which break up when $\varepsilon>0$.

In this special case, the author proposed an asymptotic expansion for the area of the main lobes of the resulting turnstile that takes the form of a power series (with even terms) in $\varepsilon$. See [Gelfreich and Simó 09, Delshams and Ramirez-Ros 99, Levallois and Tabanov 93] for more references on the computation of separatrices splitting.

In this paper our approach is experimental. We study examples and present strong numerical evidence for the following expansion of the width of the homoclinic zone:

$$
\begin{aligned}
\log \left(\nu^{+}(\mu)-\nu^{-}(\mu)\right) \asymp & \log (K(\mu, \gamma-2)) \\
& +\sum_{k \geq 1} m_{k} \mu^{k / 4}+\log \mu \sum_{k \geq 1} n_{k} \mu^{k / 2},
\end{aligned}
$$

where $K$ is given by $(1-7)$.

Remark 1.1. One easily checks that the following formula can be derived from (1-8):

$$
\nu^{+}(\mu)-\nu^{-}(\mu) \asymp K(\mu, \gamma-2) \sum_{k \geq 0} \sum_{0 \leq j \leq\left[\frac{k}{2}\right]} \tilde{c}_{k, j} \mu^{k / 4} \log ^{j} \mu,
$$

where $\left[\frac{k}{2}\right]$ stands for the integer part of $k / 2$ and the $\tilde{c}_{k, j}$ 's depend on the $m_{k}$ 's and the $n_{k}$ 's.

Observe that (1-9) is a double series with logarithmic terms, and numerically, for such an expansion, we do not know any efficient techniques to compute the corresponding coefficients with high precision. However, the asymptotic series (1-8) does not involve a double summation and therefore the corresponding coefficients can be computed with a much higher precision.

Remark 1.2. Logarithmic terms may vanish. This occurs, for instance, in the case of the Hénon map; see the next section for more details.

Remark 1.3. From the numerical data, we are able to guess a simple analytic expression for the first logarithmic term in (1-8). More precisely, we have $n_{1}=-\left(\frac{6(\gamma-2)}{7 \sqrt{2}}\right)^{2}$, which is valid for all families studied in this article.

Remark 1.4. Our computations require very high precision, of several thousand digits. For this we use the package Mathematica, which allows us to set the precision of our computation as high as required.

The paper is organized as follows. We shall consider three different families that satisfy (up to appropriate 
smooth changes of coordinates) the setting above with different nonlinear terms. As a result of our experiments, for each family we shall state the asymptotics for the width of the homoclinic zone, confirming formula (1-8).

Looking for the width of the homoclinic zone amounts to fixing one parameter, say $\mu$, in the unfolding (1-3), and finding the values of the second parameter, say $\nu$, for which the system admits a first and a last homoclinic tangency. We say that $\mu$ is the main parameter and $\nu$ is the slave parameter. In Section 3 we briefly present the strategy that we follow.

The rest of the section is devoted to the computation of the invariant (stable and unstable) manifolds at the saddle point. The splitting function, which is a key ingredient of our techniques, is presented.

Indeed, primary homoclinic orbits are in one-to-one correspondence with zeros of the splitting function. Therefore, the first and last homoclinic tangencies will correspond to double zeros of the splitting function. Moreover, the splitting function is periodic, with exponentially decreasing harmonics, and is well approximated by the splitting determinant. With good precision, computing the width of the zone amounts to the computation of the first two harmonics of the splitting function and their dependence with respect to the slave parameter (the main parameter being fixed).

For each family, we compute the width of the homoclinic zone for several hundred values of the main parameter $\mu$ and collect the results in a set of renormalized data.

In the next step, the coefficients in (1-8) (considered as an ansatz) are extracted by interpolation techniques.

The remaining part of the paper is devoted to the verification of the validity of our results. More precisely, we test the ansatz (1-8) and determine how precise our data for the width of the homoclinic zone should be in order to produce reliable results for the coefficients of the asymptotic expansion.

Finally, the constant coefficient of the expansion should coincide with the splitting constant [Gelfreich 03]: following the procedure developed in [Gelfreich and Naudot 06], we compare these constants with the constant coefficients of the expansions.

\section{MAIN RESULTS}

Before presenting our main results, we first introduce the following notions.

\subsection{Asymptotic Sequences and Expansions}

Let $\varepsilon_{0}>0$ be given and let

$$
\tilde{\mathcal{S}}=\left\{f_{0}, f_{1}, \ldots, f_{n}, \ldots\right\},
$$

where $f_{0} \equiv 1$ and for each integer $i>0, f_{i}:\left(0, \varepsilon_{0}\right) \rightarrow \mathbb{R}$ is a smooth positive function such that

$$
\lim _{x \rightarrow 0^{+}} \frac{f_{i+1}(x)}{f_{i}(x)}=0,
$$

or in other words, $f_{i+1}(x)=o\left(f_{i}(x)\right)$. Such a family $\tilde{\mathcal{S}}$ is called an asymptotic sequence.

In this paper we shall consider the following asymptotic sequences:

$$
\tilde{\mathcal{P}}=\left\{1, x, x^{2} \ldots, x^{n}, \ldots\right\}
$$

that is, $f_{i}(x)=x^{i}$ and the Dulac asymptotic sequence [Marděsic 94]

$$
\begin{aligned}
\tilde{\mathcal{D}}=\{ & 1, x, x^{2} \log x, x^{2}, x^{3}, x^{4} \log x, x^{4}, \ldots, x^{2 n} \log (x), \\
& \left.x^{2 n}, x^{2 n+1}, \ldots\right\}
\end{aligned}
$$

that is, for all integer $n \geq 0$,

$$
\begin{aligned}
f_{3 n}(x) & =x^{2 n}, \quad f_{3 n+1}(x)=x^{2 n+1}, \\
f_{3 n+2}(x) & =x^{2 n+2} \log (x) .
\end{aligned}
$$

Let $\phi:\left(0, \varepsilon_{0}\right) \rightarrow \mathbb{R}$ be a smooth function. We say that

$$
\phi(x) \asymp \sum_{n \in \mathbb{N}} \alpha_{n} f_{n}(x)
$$

is an asymptotic expansion of $\phi$ at 0 (where $\left\{f_{n}\right\}_{n \in \mathbb{N}}$ is an asymptotic sequence and all $\alpha_{n}$ 's are real) if for all integer $n$,

$$
\phi(x)-\phi^{\{n\}}(x)=\mathcal{O}\left(f_{n+1}(x)\right),
$$

where $\phi^{\{n\}}(x)=\sum_{i=0}^{n} \alpha_{i} f_{i}(x)$. With respect to expansions of the form (2-3), no convergence is implied and often the $\alpha_{i}$ 's are Gevrey-1, i.e.,

there exist $M>0, r>0$, such that for all $k \geq 0$, $\left|\alpha_{k}\right| \leq M k ! / r^{k}$.

\subsection{Quadratic Family}

Our first example is the quadratic map

$$
\begin{aligned}
\mathbf{Q} & =\mathbf{Q}_{\mu, \nu, \gamma}: \mathbb{R}^{2} \rightarrow \mathbb{R}^{2}, \\
(x, y) & \mapsto\left(x+y, y+x^{2}-\mu+\gamma x y+\nu y\right) .
\end{aligned}
$$


Observe that $\mathbf{Q}$ mimics the unfolding (1-1), i.e., it takes the form of $F_{\mu, \nu}$ and ignores the higher-order terms. We normalize the width of the homoclinic zone associated to the quadratic family by defining

$$
S_{\gamma}(\mu)=\frac{\nu^{+}(\mu)-\nu^{-}(\mu)}{K(\mu, \gamma-2)} .
$$

Within the precision of our computations we observe that

$$
\log S_{\gamma}(\mu) \asymp \sum_{k \geq 0} M_{k}(\gamma) \mu^{k / 4}+\log \mu \sum_{k \geq 1} N_{k}(\gamma) \mu^{k / 2},
$$

where $M_{k}(\gamma)$ and $N_{k}(\gamma)$ are real coefficients that depend on the parameter $\gamma$.

Comparing with (1-6), we see that

$$
\exp \left(M_{0}(\gamma)\right) \equiv \boldsymbol{\Theta}_{\gamma}
$$

is the splitting constant associated with $\mathbf{Q}_{0,0, \gamma}$. Moreover, as we announced in the previous section, we have

$$
N_{1}(\gamma) \equiv-\left(\frac{6(\gamma-2)}{7 \sqrt{2}}\right)^{2}
$$

For each value of $\gamma$, the $M_{k}$ 's and $N_{k}$ 's can be computed with very high precision; see Table 1 for an illustration.

Formula (2-5) has been verified for the first 76 coefficients: $M_{k}, k=0, \ldots, 50$, and $N_{\ell}, \ell=1, \ldots, 25$. Although the precision decreases almost linearly as $k$ and $\ell$ increase, the first 76 coefficients can be computed with 60 correct digits. To compute these first coefficients, we need to compute the width of the homoclinic zone with at least 200 correct digits; see Section 4.2 for more details.

Even if we can propose an analytic expression for $N_{1}(\gamma)$, we have not been able to guess analytic expressions for the other coefficients $N_{k}$ and $M_{k}$.

\subsection{Bogdanov Family}

Our second example is the Bogdanov map [Arrowsmith 93, Arrowsmith and Place 90, Bogdanov 75]

$$
\begin{gathered}
\mathbf{B}=\mathbf{B}_{a, b, \tilde{\gamma}}: \mathbb{R}^{2} \rightarrow \mathbb{R}^{2} \\
(x, y) \mapsto\left(x+y+x^{2}+\tilde{\gamma} x y+a x+b y,\right. \\
\left.y+x^{2}+\tilde{\gamma} x y+a x+b y\right) .
\end{gathered}
$$

The Bogdanov map, see for example [Arnol'd 72, Bogdanov 81], is the Euler map of a two-dimensional system of ordinary differential equations.

Arrowsmith studied the bifurcations and basins of attraction and showed the existence of mode locking,
Arnold tongues, and chaos [Arrowsmith 93]; see also [Arrowsmith and Place 90] for more details.

For this map the saddle point is located at the origin. This map can be transformed to the form (1-3). Indeed, let

$$
\begin{aligned}
& u=x-\frac{a}{2} \\
& v=y+\left(x-\frac{a}{2}\right)^{2}+\tilde{\gamma}\left(x-\frac{a}{2}\right) y+a\left(x-\frac{a}{2}\right)+b y .
\end{aligned}
$$

We retrieve the map (1-2) and higher-order terms (1-3) by putting

$$
\nu=a+b-(\tilde{\gamma}+2) \frac{a}{2}, \quad \gamma=\tilde{\gamma}+2, \quad \mu=a^{2} / 4,
$$

and

$$
f_{\mu, \nu}=(x+y)^{2}-\mu+\gamma y^{2} .
$$

The parameter $a$ is chosen to be the main parameter and $b$ the slave parameter. From (1-5), the Bogdanov map admits a homoclinic zone near the line

$$
b^{ \pm}(a)=\frac{6}{7} a \tilde{\gamma}+\mathcal{O}\left(a^{3 / 2}\right)
$$

The normalized width takes the form

$$
\tilde{S}_{\tilde{\gamma}}(a)=\frac{b^{+}(a)-b^{-}(a)}{K\left(a^{2} / 4, \tilde{\gamma}\right)}
$$

Similarly to the quadratic family, our experiments showed that $\log \tilde{S}_{\tilde{\gamma}}$ satisfies the following asymptotics:

$$
\log \tilde{S}_{\tilde{\gamma}}(a) \asymp \sum_{k \geq 0} A_{k}(\tilde{\gamma}) a^{k / 2}+\log a \sum_{k \geq 1} B_{k}(\tilde{\gamma}) a^{k},
$$

where $A_{k}(\tilde{\gamma})$ and $B_{k}(\tilde{\gamma})$ are real coefficients that depend on the parameter $\gamma$. Comparing with $(1-6)$, we see that

$$
\exp \left(A_{0}(\tilde{\gamma})\right) \equiv \boldsymbol{\Theta}_{\tilde{\gamma}}
$$

is the splitting constant associated with $\mathbf{B}_{0,0, \tilde{\gamma}}$. Moreover, we observe numerically that $B_{1}(\tilde{\gamma}) \equiv-(6 \tilde{\gamma} / 7)^{2}$.

In Table 1, we provide typical results for our computation for the quadratic and Bogdanov maps. Although the first 20 coefficients do not show a tendency to grow rapidly, we conjecture that the series $(2-5)$ and $(2-6)$ diverge and belong to the Gevrey-1 class defined at the end of Section 2.1; compare with [Gelfreich and Simó 09].

\subsection{Hénon Map}

The last example to be considered in this paper is defined by

$$
\mathbf{H}=\mathbf{H}_{\tilde{a}, \tilde{b}}: \mathbb{R}^{2} \rightarrow \mathbb{R}^{2}, \quad(u, v) \mapsto\left(u_{1}, v_{1}\right),
$$




\begin{tabular}{|c|c|r|}
\hline Coefficient & Scale & \multicolumn{1}{|c|}{ Value } \\
\hline$A_{0}$ & 1 & 61.26721889 \\
$A_{1}$ & $a^{1 / 2}$ & -29.82701974 \\
$B_{1}$ & $a \log a$ & -6.612244898 \\
$A_{2}$ & $a$ & 5.824479250 \\
$A_{3}$ & $a^{3 / 2}$ & 17.41183781 \\
$B_{2}$ & $a^{2} \log a$ & 5.649967276 \\
$A_{4}$ & $a^{2}$ & -0.2874798361 \\
$A_{5}$ & $a^{5 / 2}$ & -22.04012159 \\
$B_{3}$ & $a^{3} \log a$ & -6.966574583 \\
$A_{6}$ & $a^{3}$ & -6.250578833 \\
$A_{7}$ & $a^{7 / 2}$ & 39.27382902 \\
$B_{4}$ & $a^{4} \log a$ & 10.92891913 \\
$A_{8}$ & $a^{4}$ & 19.31687979 \\
$A_{9}$ & $a^{9 / 2}$ & -82.17477248 \\
$B_{5}$ & $a^{5} \log a$ & -20.01663759 \\
$A_{10}$ & $a^{5}$ & -50.35178499 \\
$A_{11}$ & $a^{11 / 2}$ & 186.9039750 \\
$B_{6}$ & $a^{6} \log a$ & 40.63376347 \\
$A_{12}$ & $a^{6}$ & 128.7996196 \\
$A_{13}$ & $a^{13 / 2}$ & -444.7385574 \\
\hline
\end{tabular}

\begin{tabular}{|c|c|r|}
\hline Coefficient & Scale & \multicolumn{1}{c|}{ Value } \\
\hline$M_{0}$ & 1 & -13.35083105 \\
$M_{1}$ & $\mu^{1 / 4}$ & -35.34533603 \\
$N_{1}$ & $\mu^{1 / 2} \log \mu$ & -9.183673469 \\
$M_{2}$ & $\mu^{1 / 2}$ & -25.71572403 \\
$M_{3}$ & $\mu^{3 / 4}$ & 60.69366755 \\
$N_{2}$ & $\mu \log \mu$ & -41.92449575 \\
$M_{4}$ & $\mu$ & -215.4221683 \\
$M_{5}$ & $\mu^{5 / 4}$ & -45.92851439 \\
$N_{3}$ & $\mu^{3 / 2} \log \mu$ & -242.5333437 \\
$M_{6}$ & $\mu^{3 / 2}$ & -960.8699623 \\
$M_{7}$ & $\mu^{7 / 4}$ & 755.3601690 \\
$N_{4}$ & $\mu^{2} \log \mu$ & -1587.303140 \\
$M_{8}$ & $\mu^{2}$ & -3308.441120 \\
$M_{9}$ & $\mu^{9 / 4}$ & 1090.837521 \\
$N_{5}$ & $\mu^{5 / 2} \log \mu$ & -11017.80445 \\
$M_{10}$ & $\mu^{5 / 2}$ & -134120.3771 \\
$M_{11}$ & $\mu^{11 / 4}$ & 22519.75418 \\
$N_{6}$ & $\mu^{3} \log \mu$ & -79363.78673 \\
$M_{12}$ & $\mu^{3}$ & 904656.6104 \\
$M_{13}$ & $\mu^{13 / 4}$ & 87833.05069 \\
\hline
\end{tabular}

TABLE 1. The first 20 coefficients of the asymptotic expansion for the Bogdanov map (left, $\tilde{\gamma}=3$ ) and the quadratic map (right, $\gamma=-3$ ). All the given digits are correct.

where

$$
u_{1}=v, \quad v_{1}=\tilde{a} v^{2}-\tilde{b} u+1 .
$$

See [Kirchgraber and Stoffer 06] for recent results concerning this family. The Hénon map has a fixed point of Bogdanov-Takens type at $\tilde{a}=\tilde{b}=1$.

We chose $\tilde{a}$ as the main parameter and $\tilde{b}$ as the slave parameter. We note that the Hénon map is conjugate to the Bogdanov family in the special case of $\tilde{\gamma}=0$. The conjugacy is given by the following change of coordinates and parameters:

$$
\begin{aligned}
& u=x, \quad v=x+y+x^{2}+a x+b y, \\
& \tilde{b}=b+1, \quad \tilde{a}=(1+b / 2)^{2}-a^{2} / 4 .
\end{aligned}
$$

We also observe that the Hénon map can be transformed to the form (1-1) with nonlinear term of the form (1-3) by putting

$$
u=\frac{1}{\tilde{a}}\left(x+\frac{\tilde{b}+1}{2}\right), \quad v=\frac{1}{\tilde{a}}\left(x+\frac{\tilde{b}+1}{2}\right)+\frac{1}{\tilde{a}} y .
$$

In the new system of coordinates, the Hénon map takes the form (1-3) with

$$
f_{\mu, \nu}=(x+y)^{2}-\mu+\nu y,
$$

where

$$
\mu=\left(1+\frac{\nu}{2}\right)^{2}-\tilde{a}, \quad \nu=\tilde{b}-1 .
$$

The Hénon map admits a homoclinic zone near the line

$$
\tilde{b}^{ \pm}(\tilde{a}) \equiv 1, \quad \tilde{a} \geq 1 .
$$

In the case of the Hénon map we define the normalized width of the zone by

$$
\tilde{S}(\tilde{a})=\frac{\tilde{b}^{+}(\tilde{a})-\tilde{b}^{-}(\tilde{a})}{K(1-\tilde{a}, 0)} .
$$

Our numerical experiments show that $\tilde{S}$ has the following asymptotic expansion:

$$
\tilde{S}(\tilde{a})=\sum_{k \geq 0} \tilde{A}_{k}(1-\tilde{a})^{k / 4} .
$$

In contrast to the case of the Bogdanov map with $\gamma \neq 2$ (i.e., $\tilde{\gamma} \neq 0$ ), the asymptotic expansion does not contain logarithmic terms. We expect this property to be closely related to the fact that the Hénon map contains a one-parameter subfamily of area-preserving maps: in [Brännström and Gelfreich 08], the authors study the exponentially small splitting of separatrices for areapreserving maps near a Hamiltonian saddle center bifurcation. This study covers the case of the area-preserving 
Hénon map. The authors show that the corresponding series does not contain logarithmic terms. In general, even when $\gamma=2$, there is no reason to expect the logarithmic terms to vanish for a map $F_{\mu, \nu}$.

\section{COMPUTING THE WIDTH OF THE HOMOCLINIC ZONE}

In this section, our approach concerns the quadratic family $\mathbf{Q}_{\mu, \nu, \gamma}$. The other families (Bogdanov and Hénon) are treated in a similar way. From now on, we do not mention the $(\mu, \nu, \gamma)$ dependencies when it is not necessary, but we may emphasize such dependence when it is needed.

\subsection{Strategy}

(i) We assume an ansatz and in particular the one given in formula (1-8);

(ii) Compute $\tilde{n}$ (several hundred) values of the width for values of $\mu^{1 / 4} \in[c, d]$, where $0<c<d$ are close to 0 (typically $c \approx 5 / 1000, d \approx 1 / 100$ ). It is convenient to work with the so-called normalized width of the homoclinic zone defined by

$$
S_{\gamma}(\mu)=\frac{\nu^{+}(\mu)-\nu^{-}(\mu)}{K(\mu, \gamma-2)}
$$

where $K$ is defined by (1-7). The result is collected in a set of data of the form

$$
\begin{aligned}
\mathcal{H}=\left\{\left(\mu_{i}^{1 / 4}, \log \left(S_{\gamma}\left(\mu_{i}\right)\right)\right),\right. & \\
& \left.c \leq \mu_{i} \leq d, i=1, \ldots, \tilde{n}\right\} .
\end{aligned}
$$

(iii) Take $\ell \in \mathbb{N}$ such that $3 \ell / 2+1 \leq \tilde{n}$ and $\ell \gg 1$ even. Then we compute the coefficients $M_{k}, k=0, \ldots, \ell$, and $N_{k}, k=1, \ldots, \ell / 2$, of the truncated expansion

$$
G^{\{3 \ell / 2\}}(\mu)=\sum_{k=0}^{\ell} M_{k}(\gamma) \mu^{k / 4}+\log \mu \sum_{k=1}^{\ell / 2} N_{k}(\gamma) \mu^{k / 2}
$$

to interpolate the set $\mathcal{H}$, i.e., for all integer $i=$ $1, \ldots, 3 \ell / 2+1$, we have

$$
\log S_{\gamma}\left(\mu_{i}\right)=\sum_{k=0}^{\ell} M_{k}(\gamma) \mu_{i}^{k / 4}+\log \mu_{i} \sum_{k=1}^{\ell / 2} N_{k}(\gamma) \mu_{i}^{k / 2} .
$$

See Section 3.10 for more details.

Remark 3.1. For the Bogdanov family, the set of data for the normalized width is denoted by

$$
\tilde{\mathcal{H}}=\left\{\left(a_{i}^{1 / 2}, \log \left(\tilde{S}_{\gamma}\left(a_{i}\right)\right)\right), \tilde{c}<a_{i}<\tilde{d}, i=1, \ldots, \tilde{n}\right\},
$$

where

$$
\tilde{S}_{\gamma}\left(a_{i}\right)=\frac{b^{+}\left(a_{i}\right)-b^{-}\left(a_{i}\right)}{K\left(a_{i}^{2} / 4, \tilde{\gamma}\right)} \text { and } \quad 0<\tilde{c}<\tilde{d}
$$

Remark 3.2. For the Hénon family, the set of data for the normalized width is denoted by

$$
\tilde{\mathcal{Z}}=\left\{\left(\left|1-\tilde{a}_{i}\right|, \tilde{S}\left(\tilde{a}_{i}\right)\right), \tilde{c}<\left|1-\tilde{a}_{i}\right|<\tilde{d}, i=1, \ldots, \tilde{n}\right\},
$$

where

$$
\tilde{S}\left(\tilde{a}_{i}\right)=\frac{\tilde{b}^{+}\left(\tilde{a}_{i}\right)-\tilde{b}^{-}\left(\tilde{a}_{i}\right)}{K\left(\left(1-\tilde{a}_{i}\right), 0\right)} \quad \text { and } \quad 0<\tilde{c}<\tilde{d} .
$$

\subsection{Invariant Manifolds}

We now compute the stable and unstable manifolds at the saddle point. In what follows, our description concerns the quadratic map $\mathbf{Q}$, but similar computations are done for the Bogdanov map and the Hénon map.

From (2-4), the map $\mathbf{Q}$ has two fixed points:

$$
\mathbf{S}_{\mu}=(\sqrt{\mu}, 0), \quad \mathbf{C}_{\mu}=(-\sqrt{\mu}, 0) .
$$

The point $\mathbf{C}_{\mu}$ is a focus and $\mathbf{S}_{\mu}$ is a saddle and will be the point of interest. The eigenvalues of $d \mathbf{Q}\left(\mathbf{S}_{\mu}\right)$ are given by

$$
\begin{aligned}
& \lambda_{1}=\frac{1}{2}\left(2+\nu+\gamma \sqrt{\mu}-\sqrt{(\gamma \sqrt{\mu}+\nu)^{2}+8 \sqrt{\mu}}\right), \\
& \lambda_{2}=\frac{1}{2}\left(2+\nu+\gamma \sqrt{\mu}+\sqrt{(\gamma \sqrt{\mu}+\nu)^{2}+8 \sqrt{\mu}}\right) .
\end{aligned}
$$

For $\mu>0$ sufficiently small it is clear that $\lambda_{1}<1<\lambda_{2}$.

At the saddle $\mathbf{S}_{\mu}$, the Taylor expansion of the local stable manifold $W_{\mathrm{loc}}^{s}$ and that of the local unstable manifold $W_{\text {loc }}^{u}$ are computed as follows. Denote by

$$
\begin{aligned}
\Phi_{s}:(\mathbb{R}, 0) & \rightarrow\left(\mathbb{R}^{2}, \mathbf{S}_{\mu}\right), \\
z & \mapsto \Phi_{s}(z)=\left(\sqrt{\mu}+\sum_{k=1}^{\infty} \varphi_{k} z^{k}, \sum_{k=1}^{\infty} \psi_{k} z^{k}\right),
\end{aligned}
$$

and

$$
\begin{aligned}
\Phi_{u}:(\mathbb{R}, 0) & \rightarrow\left(\mathbb{C}^{2}, \mathbf{S}_{\mu}\right), \\
z & \mapsto \Phi_{u}(z)=\left(\sqrt{\mu}+\sum_{k=1}^{\infty} f_{k} z^{k}, \sum_{k=1}^{\infty} p_{k} z^{k}\right),
\end{aligned}
$$

the parameterizations that respectively satisfy

$$
\Phi_{s}\left(\lambda_{1} z\right)=\mathbf{Q} \circ \Phi_{s}(z) \text { and } \Phi_{u}\left(\lambda_{2} z\right)=\mathbf{Q} \circ \Phi_{u}(z)
$$


for all $z$ near 0 . Substituting the series into (3-4) and collecting terms of the same order in $z$, we get, for $k \geq 1$,

$$
\begin{aligned}
\varphi_{k}+\psi_{k} & =\lambda_{1}^{k} \varphi_{k}, \\
\sum_{j=0}^{k} \varphi_{j} \varphi_{k-j}+\gamma \sum_{j=0}^{k} \varphi_{j} \psi_{k-j}+\nu \psi_{k} & =\lambda_{1}^{k} \psi_{k},
\end{aligned}
$$

and

$$
\begin{aligned}
p_{k}+f_{k} & =\lambda_{2}^{k} p_{k}, \\
\sum_{j=0}^{k} f_{j} f_{k-j}+\gamma \sum_{j=0}^{k} f_{j} p_{k-j}+\nu p_{k} & =\lambda_{2}^{k} p_{k} .
\end{aligned}
$$

Since $\lambda_{2}>1$ and $\mathbf{Q}$ is entire, from (3-4) we easily deduce that the radius of convergence of the series defined in (3-6) is infinite. Denote by $\varrho$ the radius of convergence of the series defined in (3-5). We fix $N_{\max } \in \mathbb{N}$. Since we are after a single branch of the stable manifold, we write

$$
W_{\mathrm{loc}}^{s} \approx W_{N_{\max }}^{s}=\left\{\Phi_{s, N_{\max }}(z), 0 \leq z \leq \delta_{s}\right\},
$$

where

$$
\Phi_{s, N_{\max }}(z)=\left(\sqrt{\mu}+\sum_{k=1}^{N_{\max }} \varphi_{k} z^{k}, \sum_{k=1}^{N_{\max }} \psi_{k} z^{k}\right)
$$

and where $0<\delta_{s}<\varrho$.

We proceed in the same way for the local unstable manifold, i.e.,

$$
W_{\mathrm{loc}}^{u} \approx W_{N_{\max }}^{u}=\left\{\Phi_{u, N_{\max }}(z), 0 \leq z \leq \delta_{u}\right\},
$$

where

$$
\Phi_{u, N_{\max }}(z)=\left(\sqrt{\mu}+\sum_{k=1}^{N_{\max }} f_{k} z^{k}, \sum_{k=1}^{N_{\max }} p_{k} z^{k}\right),
$$

and where $0<\delta_{u} \ll 1$.

The local invariant manifolds are computed with the following precision:

$$
\begin{aligned}
\left\|\Phi_{s, N_{\max }}(z)-\Phi_{s}(z)\right\| & =\mathcal{O}\left(z^{N_{\max }}\right), \\
\left\|\Phi_{u, N_{\max }}(z)-\Phi_{u}(z)\right\| & =\mathcal{O}\left(z^{N_{\max }}\right) .
\end{aligned}
$$

In particular, we have

$$
\left\|\Phi_{u}\left(\lambda_{2} z\right)-\mathbf{Q} \circ \Phi_{u, N_{\max }}(z)\right\|=\mathcal{O}\left(z^{N_{\max }}\right) .
$$

Since we need to study the map when homoclinic orbits are present, we need a good estimate of the global unstable manifold.

Recall that $\Phi_{u}$ is entire and therefore both components defined in (3-7) converge for all $z$ as $N_{\max } \rightarrow \infty$. However, for large $z$, the computation of the unstable manifold requires too many coefficients, and therefore (3-7) is not very convenient. We then proceed as follows.
Let $\mathrm{P}_{0}=\Phi_{u}\left(z_{0}\right) \in W^{u}$ and choose $m_{0}$ such that

$$
z_{1}=\lambda_{2}^{-m_{0}} z_{0} \leq \delta_{u}
$$

Then for any fixed $m_{0}$, we have

$$
P_{0}=\lim _{N_{\max } \rightarrow \infty} \mathbf{Q}^{m_{0}} \circ \Phi_{u, N_{\max }}\left(z_{1}\right),
$$

and if $z_{1} \ll 1$, the convergence is fast.

Therefore, by putting

$W^{u} \approx W_{N_{\max }, m}^{u}=\left\{\mathbf{Q}^{m} \circ \Phi_{u, N_{\max }}\left(\lambda_{2}^{-m} z\right), 0 \leq z \leq z_{0}\right\}$,

$m \geq m_{0}$, we get an accurate estimate of the global unstable manifold.

\subsection{Jacobian and Wronskian Functions}

Before introducing the splitting function that will play a key role in the paper, we need to introduce two additional functions.

We first define

$$
J: \mathcal{D} \rightarrow \mathbb{C}, \quad z \mapsto \operatorname{det} d \mathbf{Q}\left(\Phi_{s}(z)\right),
$$

as the Jacobian of the map $\mathbf{Q}$ along the stable manifold

$$
\Phi_{s}(z)=\left(\Phi_{s, x}(z), \Phi_{s, y}(z)\right) .
$$

A straightforward computation gives

$$
J(z)=1+\nu+(\gamma-2) \Phi_{s, x}(z)-\gamma \Phi_{s, y}(z) .
$$

In terms of series, from (3-9) we get

$$
J(z)=\sum_{k=0}^{\infty} J_{k} z^{k},
$$

where $J_{0}=1+\nu+(\gamma-2) \sqrt{\mu}$ and for all $k>0, J_{k}=$ $(\gamma-2) \phi_{k}-\gamma \psi_{k}$. The Wronskian function (along the local stable manifold)

$$
\Omega: \mathcal{D} \rightarrow \mathbb{R}, \quad z \mapsto \Omega(z),
$$

satisfies

$$
\Omega\left(\lambda_{1} z\right)=J(z) \Omega(z) .
$$

We put $\Omega_{0}=1$ and look for a solution of $(3-11)$ of the form

$$
\Omega(z)=z^{\log J_{0} / \log \lambda_{1}}\left(1+\sum_{k=1}^{\infty} \Omega_{k} z^{k}\right) .
$$

With (3-11), (3-10), and (3-12), it follows that

$$
\Omega_{n}=\frac{1}{\lambda_{1}-J_{0}}\left(J_{n}+\sum_{j=0}^{n-1} \Omega_{j} J_{n-1-j}\right) .
$$


Both series (3-10) and (3-12) are convergent. The functions $J$ and $\Omega$ will be approximated by

$$
J_{N_{\max }}(z)=\sum_{k=0}^{N_{\max }} J_{k} z^{k}
$$

and

$$
\Omega_{N_{\max }}(z)=z^{\log J_{0} / \log \lambda_{1}}\left(1+\sum_{k=1}^{N_{\max }} \Omega_{k} z^{k}\right)
$$

respectively. In this way, we have

$$
\left|\Omega_{N_{\max }}\left(\lambda_{1} z\right)-J_{N_{\max }}(z) \Omega_{N_{\max }}(z)\right|=\mathcal{O}\left(|z|^{N_{\max }}\right) .
$$

\subsection{Splitting Function and Flow Box Theorem}

In this section, we introduce the key part of our techniques. Recall that in our investigation for the width of the homoclinic zone, we fix the value of the main parameter and look for values $\nu^{+}$and $\nu^{-}$of the slave parameter that correspond, respectively, to the first and the last homoclinic tangencies. In order to find a homoclinic point we need to adjust the slave parameter in such a way that two curves on the plane have an intersection. Finding a homoclinic tangency requires additional adjustments to make this intersection degenerate. This problem is much easier in the discrete flow box coordinates, in which the stable curve coincides with the horizontal axis and the unstable one is a graph of a periodic function. A further simplification will be achieved by observing that this periodic function is very close to a trigonometric polynomial of the first order.

The splitting function $\Theta=\Theta_{\mu, \nu}$ we shall introduce now is such that the first and the last tangencies correspond to double zeros of $\Theta_{\mu, \nu^{+}}$and $\Theta_{\mu, \nu^{-}}$respectively. Our investigation amounts then to finding values $\nu^{+}$and $\nu^{-}$such that $\Theta_{\mu, \nu^{+}}$and $\Theta_{\mu, \nu^{-}}$possess double zeros.

In this section, we present the splitting function $\Theta_{\mu, \nu}$ for the quadratic map. In the case of the Bogdanov map, the splitting function is denoted by $\Theta_{a, b}$. In what follows, we assume that the parameter $(\mu, \nu)$ is such that the map $\mathbf{Q}$ possesses a homoclinic orbit, i.e., the unstable manifold intersects the local stable manifold at a point $\Phi_{u}\left(z_{u}\right)=q_{0}=\Phi_{s}\left(z_{s}\right)$. Then we fix a neighborhood $\mathcal{U}$ of the point $q_{0}$. We parameterize $W_{\text {loc }}^{s}$ near $q_{0}$ by

$$
\Gamma_{s}: \mathbb{I}_{0} \mapsto \Phi_{s}\left(z_{s} \cdot \lambda_{1}^{t}\right),
$$

where $\mathbb{I}_{0}=(-1,1)$ and $W^{u}$ near $q_{0}$ by

$$
\Gamma_{u}: \mathbb{I}_{0} \mapsto \Phi_{u}\left(z_{u} \cdot \lambda_{2}^{t}\right) .
$$

Now we state the following (flow box) lemma [Gelfreich 96].

Lemma 3.3. There exist $E_{0}>0$ and an analytic diffeomorphism

$$
\begin{aligned}
\Psi:\left(-E_{0}, E_{0}\right) \times \mathbb{I}_{0} & \rightarrow \mathbb{R}^{2}, \\
(E, t) & \mapsto \Psi(E, t)=(X(E, t), Y(E, t)),
\end{aligned}
$$

such that the following hold:

(i) $\Psi(E, t+1)=\mathbf{Q} \circ \Psi(E, t)$;

(ii) $\Psi(0,0)=q_{0}, \Psi(0, t) \in W_{\text {loc }}^{s}$ for $t \in \mathbb{I}_{0}$;

(iii) the Jacobian matrix

$$
d \Psi(E, t)=\left(\begin{array}{ll}
\partial X / \partial E & \partial X / \partial t \\
\partial Y / \partial E & \partial Y / \partial t
\end{array}\right)
$$

is such that the second column of $d \Psi(0, t)$ is $\dot{\boldsymbol{\Gamma}}_{s}=d \boldsymbol{\Gamma}_{s}(t) / d t ;$

(iv) the map $\hat{\Omega}(E, t)=\operatorname{det} d \Psi(E, t)$ satisfies $\hat{\Omega}(0, t)=$ $\Omega\left(z_{s} \cdot \lambda_{1}^{t}\right)$.

The splitting function, denoted by $\Theta_{\mu, \nu}(t)$, is the first component of

$$
\Psi^{-1} \circ \boldsymbol{\Gamma}_{u}(t)-\Psi^{-1} \circ \boldsymbol{\Gamma}_{s}(t) .
$$

Applying Taylor's theorem to the stable manifold, we get

$$
\begin{aligned}
\Psi^{-1} & \circ \boldsymbol{\Gamma}_{u}(t)-\Psi^{-1} \circ \boldsymbol{\Gamma}_{s}(t) \\
= & d \Psi^{-1}(\Psi(0, t)) \cdot\left(\boldsymbol{\Gamma}_{u}(t)-\boldsymbol{\Gamma}_{s}(t)\right) \\
& +\mathcal{O}\left(\left\|\boldsymbol{\Gamma}_{u}(t)-\boldsymbol{\Gamma}_{s}(t)\right\|^{2}\right) .
\end{aligned}
$$

The following properties hold:

- Let $0<\tilde{\delta}<\pi$. The map $\Theta_{\mu, \nu}$ has an analytic continuation onto the rectangle

$$
\begin{gathered}
\mathrm{B}=\left\{t \in \mathbb{C}\left|t=t^{\prime}+i t^{\prime \prime}, t^{\prime} \in \mathbb{I}_{0},\right| t^{\prime \prime} \mid \leq \varrho,\right. \\
\left.|\varrho|<(\pi-\tilde{\delta}) /\left|\log \lambda_{1}\right|\right\} .
\end{gathered}
$$

The function $\Theta_{\mu, \nu}$ is periodic, so we can expand it into a Fourier series:

$$
\Theta_{\mu, \nu}(t)=\sum_{j=-\infty}^{\infty} \mathbf{P}_{j}(\mu, \nu) e^{2 i \pi t} .
$$

As usual, the Fourier coefficients are defined by an integral:

$$
\mathbf{P}_{k}(\mu, \nu)=\int_{0}^{1} \Theta_{\mu, \nu}(t) e^{-2 i k \pi t} d t, \quad \text { for each } k \in \mathbb{N} .
$$


Let $0<\varrho<(\pi-\tilde{\delta}) /\left|\log \left(\lambda_{1}\right)\right|$. Since the integral of $\Theta_{\mu, \nu}(t) e^{-2 i k \pi t}$ over the boundary of the rectangle $\left\{\left(t^{\prime}+i t^{\prime \prime}\right) \mid 0 \leq t^{\prime} \leq 1,0 \leq t^{\prime \prime} \leq \varrho\right\}$ vanishes, we conclude that

$$
\begin{aligned}
& \int_{0}^{1} \Theta_{\mu, \nu}(t) e^{-2 i k \pi t} d t \\
& \quad=e^{-2 k \pi \varrho} \int_{0}^{1} \Theta_{\mu, \nu}(t+i \varrho) e^{-2 i k \pi t} d t .
\end{aligned}
$$

Consequently,

$$
\left|\mathbf{P}_{k}(\mu, \nu)\right| \leq \sup _{t \in \mathbb{I}_{0}}\left|\Theta_{\mu, \nu}(t+i \varrho)\right| \cdot e^{-2|k| \pi \varrho},
$$

i.e., the harmonics of $\Theta_{\mu, \nu}$ decrease exponentially. The function $\Theta_{\mu, \nu}$ can be well approximated by the sum of the zero- and first-order harmonics:

$$
\begin{aligned}
\Theta_{\mu, \nu}(t)= & \mathbf{P}_{-1}(\mu, \nu) e^{-2 i \pi t}+\mathbf{P}_{0}(\mu, \nu) \\
& +\mathbf{P}_{1}(\mu, \nu) e^{2 i \pi t}+\mathcal{O}_{2}(t),
\end{aligned}
$$

or equivalently, $\Theta_{\mu, \nu}$ is well approximated by a trigonometric polynomial function

$$
\begin{aligned}
\Theta_{\mu, \nu}(t)= & \mathbf{P}_{0}(\mu, \nu) \\
& +2\left|\mathbf{P}_{-1}(\mu, \nu)\right| \cos \left(2 \pi t+\arg \left(\mathbf{P}_{-1}(\mu, \nu)\right)\right) \\
& +\mathcal{O}_{2}(t),
\end{aligned}
$$

where

$$
\sup _{t \in \mathbb{I}_{0}}\left|\mathcal{O}_{2}\right|(t)=\mathcal{O}\left(e^{-4 \pi \varrho}\right) .
$$

- Since $d \Psi^{-1}(\Psi(0, t))=(d \Psi(0, t))^{-1}$, we have

$$
d \Psi^{-1}(\Psi(0, t))=\frac{1}{\hat{\Omega}(0, t)}\left(\begin{array}{cc}
\partial Y / \partial t & -\partial X / \partial t \\
\partial X / \partial E & \partial Y / \partial E
\end{array}\right) .
$$

Furthermore,

$$
\begin{aligned}
& \Psi^{-1}\left(\Gamma^{u}(t)\right)=\left(E_{u}(t), T_{u}(t)\right), \\
& \Psi^{-1}\left(\Gamma^{s}(t)\right)=\left(E_{s}(t), T_{s}(t)\right)=(0, t),
\end{aligned}
$$

with (3-14) and (3-15). It follows that

$$
\begin{aligned}
\Theta_{\mu, \nu}(t)= & E_{u}(t)-E_{s}(t) \\
= & \frac{1}{\hat{\Omega}(0, t)} \operatorname{det}\left(\frac{d}{d t} \boldsymbol{\Gamma}_{s}(t), \boldsymbol{\Gamma}_{u}(t)-\boldsymbol{\Gamma}_{s}(t)\right) \\
& +\mathcal{O}\left(\left\|\boldsymbol{\Gamma}_{u}(t)-\boldsymbol{\Gamma}_{s}(t)\right\|^{2}\right) .
\end{aligned}
$$

Thus we obtain a formula suitable for computation of the splitting function in terms of the parameterization of the stable and unstable manifolds:

$$
\Theta_{\mu, \nu}(t)=\tilde{\Theta}_{\mu, \nu}(t)+\tilde{h}_{\mu, \nu}(t),
$$

where

$$
\tilde{\Theta}_{\mu, \nu}(t)=\frac{1}{\Omega\left(z_{s} \cdot \lambda_{1}^{t}\right)} \operatorname{det}\left(\frac{d}{d t} \boldsymbol{\Gamma}_{s}(t), \boldsymbol{\Gamma}_{u}(t)-\boldsymbol{\Gamma}_{s}(t)\right)
$$

is the splitting determinant and

$$
\left|\tilde{h}_{\mu, \nu}(t)\right|=\mathcal{O}\left(\sup _{t \in \mathbb{I}_{0}}\left|\tilde{\Theta}_{\mu, \nu}(t)\right|^{2}\right) .
$$

Note that even if the invariant manifolds and the Wronskian are computed with a very high precision, the function $\Theta_{\mu, \nu}(t)$ is evaluated only with a relative error of order $\mathcal{O}\left(\sup _{t \in \mathbb{I}_{0}}\left|\Theta_{\mu, \nu}\right|\right)$.

\subsection{Approaching a Primary Homoclinic Orbit}

In order to compute the width of the homoclinic zone, we first find a value $\nu=\bar{\nu}$ where the map possesses a primary homoclinic orbit. Near $\nu=\bar{\nu}$, Lemma 3.3 will then be applied, and the splitting determinant $\tilde{\Theta}_{\mu, \nu}$ will be computed. We proceed as follows: We fix $0<z_{s}<\delta_{s}$ and a section $\Sigma$ transverse to the local stable manifold at $p_{\nu}=\Phi_{s}\left(z_{s}\right)$. We parameterize $\Sigma$ as follows:

$$
\Sigma=\left\{p_{\nu}+(0, y),-y_{0}<y<y_{0}\right\},
$$

where $0<y_{0} \ll 1$. For each value of the main parameter, we consider the slave parameter close to

$$
\nu_{0}=\left(\frac{5(\gamma-2)}{7}\right) \sqrt{\mu}
$$

and compute a point $q_{\nu} \in W^{u} \cap \Sigma$ that is the first intersection of $W^{u}$ with the section.

In order to increase the speed of computations, we use Newton's method to solve the equation $\boldsymbol{\Gamma}_{u}(t) \in \Sigma$. After that we adjust $\nu$ in such a way that $q_{\nu}=p_{\nu}$. We do not know an easy way to evaluate the derivative of $q_{\nu}$ with respect to $\nu$. Therefore we cannot apply Newton's method. However, we replace the derivative by a finite difference approximation and use the secant method. In other words, we consider the limit of the following sequence:

$$
\nu_{n+1}=\nu_{n}+\frac{\bar{\delta} y_{\nu_{n}}}{y_{\nu_{n}+\bar{\delta}}-y_{\nu_{n}}},
$$

where $q_{\nu}=p_{\nu}+\left(0, y_{\nu}\right)$ and where $0<\bar{\delta} \ll 1$. Set

$$
\bar{\nu}=\lim _{n \rightarrow \infty} \nu_{n} .
$$

Since $p_{\bar{\nu}}=q_{\bar{\nu}}$, the point $(\mu, \bar{\nu})$ belongs to the homoclinic zone.

Our next step is with the computation of the width $\nu^{+}(\mu)-\nu^{-}(\mu)$ for the given value of $\mu$. The zeros (and 
double zeros) of $\Theta_{\mu, \nu}$ are in one-to-one correspondence with primary homoclinic orbits (and homoclinic tangencies) for the corresponding map; see [Gelfreich 96, Gelfreich 03] for more details. We then replace the problem of finding homoclinic points and homoclinic tangencies to that of finding double zeros of the splitting function $\Theta_{\mu, \nu}$.

\subsection{First and Last Tangencies}

The most natural way to compute the width of the homoclinic zone is to estimate both $\nu^{+}=\nu^{+}(\mu)$ and $\nu^{-}=\nu^{-}(\mu)$. Write

$$
\Theta_{\mu, \nu}(t)=\mathbf{P}_{0}(\mu, \nu)+\hat{\Theta}_{\mu, \nu}(t) .
$$

At the first tangency $\left(\nu=\nu^{-}\right)$, the graph of the splitting function is located below the $t$-axis, and $\Theta_{\mu, \nu^{-}}$admits a double zero. Therefore there exists $t^{-} \in \mathbb{I}_{0}$ such that

$$
\begin{aligned}
\Theta_{\mu, \nu^{-}}\left(t^{-}\right) & =\sup _{t \in \mathbb{I}_{0}} \Theta_{\mu, \nu^{-}}(t)=0 \\
& =\mathbf{P}_{0}\left(\mu, \nu^{-}\right)+\sup _{t \in \mathbb{I}_{0}} \hat{\Theta}_{\mu, \nu^{-}}(t) .
\end{aligned}
$$

At the last tangency $\left(\nu=\nu^{+}\right)$the graph of the splitting function is located above the $t$-axis, and $\Theta_{\mu, \nu^{+}}$admits a double zero.

Therefore there exists $t^{+} \in \mathbb{I}_{0}$ such that

$$
\begin{aligned}
\Theta_{\mu, \nu^{+}}\left(t^{+}\right) & =\inf _{t \in \mathbb{I}_{0}} \Theta_{\mu, \nu^{+}}(t)=0 \\
& =\mathbf{P}_{0}\left(\mu, \nu^{+}\right)+\inf _{t \in \mathbb{I}_{0}} \hat{\Theta}_{\mu, \nu^{+}}(t) .
\end{aligned}
$$

If we neglect $\mathcal{O}_{2}$ in (3-20), then (3-27) and (3-28) are equivalent to

$$
\begin{aligned}
& \mathbf{P}_{0}\left(\mu, \nu^{+}\right)-2\left|\mathbf{P}_{-1}\left(\mu, \nu^{+}\right)\right|=0, \\
& \mathbf{P}_{0}\left(\mu, \nu^{-}\right)+2\left|\mathbf{P}_{-1}\left(\mu, \nu^{-}\right)\right|=0 .
\end{aligned}
$$

In this way the problem of finding the first and the last tangencies is replaced by scalar equations in one variable each. Therefore, instead of looking for intersections between $W_{\text {loc }}^{u}$ and $W^{u}$ and their tangencies, we save considerable time by simply solving a scalar equation. Observe that for $\nu$ near $\bar{\nu}$, for all $t \in \mathbb{I}_{0}$ we have

$$
\begin{aligned}
\mathbf{P}_{0}(\mu, \nu) & =\mathcal{O}\left(\mid \mathbf{P}_{1}(\mu, \nu)\right), \\
\sup _{t \in \mathbb{I}_{0}}\left|\Theta_{\mu, \nu}(t)\right| & =\mathcal{O}\left(\mid \mathbf{P}_{1}(\mu, \nu)\right) .
\end{aligned}
$$

From (3-19) we need only four points per period to evaluate $\mathbf{P}_{0}$ and $\mathbf{P}_{ \pm 1}$.
Concretely, we write

$$
\begin{aligned}
\mathbf{P}_{0}(\mu, \nu) \approx & \mathbf{R}_{0}(\mu, \nu)=\frac{1}{2}\left(\tilde{\Theta}_{\mu, \nu}(0)+\tilde{\Theta}_{\mu, \nu}\left(\frac{1}{2}\right)\right) \\
\mathbf{P}_{-1}(\mu, \nu) \approx & \mathbf{R}_{-1}(\mu, \nu) \\
= & \frac{1}{4}\left(\tilde{\Theta}_{\mu, \nu}(0)-\tilde{\Theta}_{\mu, \nu}\left(\frac{1}{2}\right)\right. \\
& \left.\quad+i\left(\tilde{\Theta}_{\mu, \nu}\left(\frac{1}{4}\right)-\Theta_{\mu, \nu}\left(\frac{-1}{4}\right)\right)\right) \\
\mathbf{P}_{1}(\mu, \nu) \approx & \mathbf{R}_{1}(\mu, \nu) \\
= & \frac{1}{4}\left(\tilde{\Theta}_{\mu, \nu}(0)-\tilde{\Theta}_{\mu, \nu}\left(\frac{1}{2}\right)\right. \\
& \left.\quad-i\left(\tilde{\Theta}_{\mu, \nu}\left(\frac{1}{4}\right)-\tilde{\Theta}_{\mu, \nu}\left(\frac{-1}{4}\right)\right)\right) .
\end{aligned}
$$

From (3-19), thanks to (3-23) and (3-25), the approximation here means that

$$
\begin{aligned}
& \max \left\{\left|\mathbf{R}_{0}(\mu, \nu)-\mathbf{P}_{0}(\mu, \nu)\right|,\left|\mathbf{R}_{ \pm 1}(\mu, \nu)-\mathbf{P}_{ \pm 1}(\mu, \nu)\right|\right\} \\
& \quad=\mathcal{O}\left(\sup _{t \in \mathbb{I}_{0}}\left|\Theta_{\mu, \nu}(t)\right|^{2}\right) .
\end{aligned}
$$

Moreover, with (3-30) we have

$$
\left|\mathbf{R}_{ \pm 1}(\mu, \nu)-\mathbf{P}_{ \pm 1}(\mu, \nu)\right|=\mathcal{O}\left(\left|\mathbf{R}_{ \pm 1}(\mu, \nu)\right|^{2}\right) .
$$

We then solve

$$
\begin{aligned}
& \mathbf{R}_{0}\left(\mu, \tilde{\nu}^{+}\right)-2\left|\mathbf{R}_{-1}\left(\mu, \tilde{\nu}^{+}\right)\right|=0 \\
& \mathbf{R}_{0}\left(\mu, \tilde{\nu}^{-}\right)+2\left|\mathbf{R}_{-1}\left(\mu, \tilde{\nu}^{-}\right)\right|=0 .
\end{aligned}
$$

From (3-30), (3-32), (3-33), and (3-34), we have

$$
\begin{aligned}
& \Theta_{\mu, \tilde{\nu}^{-}}\left(t^{-}\right)=\mathcal{O}\left(\mathbf{R}_{-1}^{2}\left(\mu, \tilde{\nu}^{-}\right)\right), \\
& \Theta_{\mu, \tilde{\nu}^{+}}\left(t^{+}\right)=\mathcal{O}\left(\mathbf{R}_{-1}^{2}\left(\mu, \tilde{\nu}^{+}\right)\right) .
\end{aligned}
$$

Let $t_{0} \in \mathbb{I}_{0}$. By the mean value theorem, we have

$$
\begin{aligned}
& \left|\nu^{+}-\tilde{\nu}^{+}\right|=\mathcal{O}\left(\frac{\mathbf{R}_{-1}^{2}(\mu, \bar{\nu})}{\partial \Theta_{\mu, \nu}\left(t_{0}\right) /\left.\partial \nu\right|_{\nu=\bar{\nu}}}\right), \\
& \left|\nu^{-}-\tilde{\nu}^{-}\right|=\mathcal{O}\left(\frac{\mathbf{R}_{-1}^{2}(\mu, \bar{\nu})}{\partial \Theta_{\mu, \nu}\left(t_{0}\right) /\left.\partial \nu\right|_{\nu=\bar{\nu}}}\right) .
\end{aligned}
$$

This approach gives a good estimate of the locus of the homoclinic zone and therefore of the corresponding width, but requires the computation of both $\nu^{+}$and $\nu^{-}$ to very high precision. To be more precise, assume that we want to compute the width of the homoclinic zone for a given value of the main parameter with $N$ correct digits, while the width of the zone (roughly estimated with formula (1-6)) satisfies

$$
10^{-N_{z}-1} \leq \nu^{+}-\nu^{-}<10^{-N_{z}},
$$


where $N_{z} \gg 1$. Thus we need to compute both $\nu^{+}$and $\nu^{-}$with $N_{z}+N$ correct digits. We observe (numerically) that

$$
\tilde{\nu}^{+}-\tilde{\nu}^{-}=\mathcal{O}\left(\frac{\left|\mathbf{R}_{-1}\right|(\mu, \bar{\nu})}{\partial \Theta_{\mu, \nu}\left(t_{0}\right) /\left.\partial \nu\right|_{\nu=\bar{\nu}}}\right)
$$

also compare with (3-42) below.

Therefore with (3-36) and (3-38), $\tilde{\nu}^{+}-\tilde{\nu}^{-}$gives an estimate of the width with a relative error of the same order. In particular, this means that we cannot choose $N$ bigger than $N_{z}$. With this method, thanks to (3-33), the estimates of $\mathbf{P}_{0}(\mu, \nu)$ and of $\mathbf{P}_{-1}(\mu, \nu)$ are obtained with a relative error of the same order as $\left|\mathbf{P}_{-1}(\mu, \bar{\nu})\right|$. This requires the computation of the splitting determinant with the same relative precision. When the main parameter tends to 0 , since the eigenvalues $\lambda_{1}$ and $\lambda_{2}$ tend to 1 , the number of iterations (i.e., $m_{0}$ ) and the number of terms in $(3-7)$ (i.e., $\left.N_{\max }\right)$ required to compute the unstable manifold need to be chosen bigger and bigger.

Moreover, in order to guarantee (3-33), we need to have $\mathbf{P}_{0}(\mu, \nu)=\mathcal{O}\left(\mathbf{P}_{-1}(\mu, \nu)\right)$, i.e., (3-30), which requires that the local stable and unstable manifolds be close to one another, and more precisely, that

$$
\left\|\boldsymbol{\Gamma}_{u}(t)-\boldsymbol{\Gamma}_{s}(t)\right\|=\mathcal{O}(K(\mu, \gamma-2)) .
$$

As a conclusion, as the main parameter tends to 0 , this approach becomes more and more delicate.

In what follows, we propose another approach, one that does not require the computation of $\mathbf{P}_{0}(\mu, \nu)$, yet still requires a first value of $\nu=\bar{\nu}$ such that $(3-30)$ and gives an estimate of the width with the same precision.

\subsection{The Real Approach}

From (3-27) and (3-28) we have

$\mathbf{P}_{0}\left(\mu, \nu^{+}\right)-\mathbf{P}_{0}\left(\mu, \nu^{-}\right)=-\inf _{t \in \mathbb{I}_{0}} \hat{\Theta}_{\mu, \nu^{+}}(t)+\sup _{t \in \mathbb{I}_{0}} \hat{\Theta}_{\mu, \nu^{-}}(t)$.

Furthermore, from the mean value theorem, there exists $\nu^{-} \leq \nu_{2} \leq \nu^{+}$such that

$$
\mathbf{P}_{0}\left(\mu, \nu^{+}\right)-\mathbf{P}_{0}\left(\mu, \nu^{-}\right)=\left.\frac{\partial \mathbf{P}_{0}}{\partial \nu}\right|_{\nu=\nu_{2}} \cdot\left(\nu^{+}-\nu^{-}\right) .
$$

Thus we get

$$
\nu^{+}-\nu^{-}=\frac{\sup _{t \in \mathbb{I}_{0}} \hat{\Theta}_{\mu, \nu^{-}}(t)-\inf _{t \in \mathbb{I}_{0}} \hat{\Theta}_{\mu, \nu^{+}}(t)}{\partial \mathbf{P}_{0} /\left.\partial \nu\right|_{\nu=\nu_{2}}} .
$$

We observe (numerically) that $\hat{\Theta}_{\mu, \nu}$ does not change much with respect to $\nu$. More precisely, for all $\nu^{-} \leq \nu_{3} \leq$ $\nu^{+}, \nu^{-} \leq \nu_{4} \leq \nu^{+}$, and for all $t \in \mathbb{I}_{0}$,

$$
\frac{\left|\hat{\Theta}_{\mu, \nu_{4}}(t)-\hat{\Theta}_{\mu, \nu_{3}}(t)\right|}{\nu_{4}-\nu_{3}}=\mathcal{O}\left(\left|\mathbf{P}_{-1}\right|(\mu, \bar{\nu})\right) .
$$

Thus, with (3-20) and (3-30) we have

$$
\begin{aligned}
& \sup _{t \in \mathbb{I}_{0}} \Theta_{\mu, \nu^{-}}(t)-\inf _{t \in \mathbb{I}_{0}} \Theta_{\mu, \nu^{+}}(t) \\
& \quad=4\left|\mathbf{P}_{-1}\right|(\mu, \bar{\nu})+\mathcal{O}\left(\mathbf{P}_{-1}^{2}(\mu, \bar{\nu})\right) .
\end{aligned}
$$

Furthermore, with (3-26) we have

$$
\begin{aligned}
\left.\frac{\partial \mathbf{P}_{0}}{\partial \nu}\right|_{\nu=\nu_{2}}(t) & =\left.\frac{\partial \Theta_{\mu, \nu}}{\partial \nu}\right|_{\nu=\nu_{2}}(t)-\left.\frac{\partial \hat{\Theta}_{\mu, \nu}}{\partial \nu}\right|_{\nu=\nu_{2}}(t) \\
& =\left.\frac{\partial \Theta_{\mu, \nu}}{\partial \nu}\right|_{\nu=\nu_{2}}(t)+\mathcal{O}\left(\mathbf{P}_{-1}(\mu, \bar{\nu})\right) .
\end{aligned}
$$

With (3-22) and (3-23) we have

$$
\left.\frac{\partial \Theta_{\mu, \nu}}{\partial \nu}\right|_{\nu=\nu_{2}}(t)=\left.\frac{\partial \tilde{\Theta}_{\mu, \nu}}{\partial \nu}\right|_{\nu=\nu_{2}}(t)+\mathcal{O}\left(\tilde{\Theta}_{\mu, \bar{\nu}}(t)\right) .
$$

We then write

$$
\begin{aligned}
\tilde{\Theta}_{\mu, \nu_{4}}(t)-\tilde{\Theta}_{\mu, \nu_{3}}(t)= & \left.\frac{\partial \tilde{\Theta}_{\mu, \nu}}{\partial \nu}(t)\right|_{\nu=\nu_{3}} \cdot\left(\nu_{4}-\nu_{3}\right) \\
& +\mathcal{O}\left(\left(\nu_{4}-\nu_{3}\right)^{2}\right)
\end{aligned}
$$

and therefore

$$
\frac{\tilde{\Theta}_{\mu, \nu_{4}}(t)-\tilde{\Theta}_{\mu, \nu_{3}}(t)}{\nu_{4}-\nu_{3}}=\left.\frac{\partial \tilde{\Theta}_{\mu, \nu}}{\partial \nu}(t)\right|_{\nu=\nu_{2}}+\mathcal{O}\left(\left(\nu^{+}-\nu^{-}\right)\right) .
$$

We observe (numerically) that the left-hand side of (3-48) stays away from 0 as the main parameter tends to 0 . More precisely, there exists $v_{0}>0$ such that for all $\mu>0, \nu_{3}, \nu_{4}$ near $\bar{\nu}$ and for all $t \in \mathbb{I}_{0}$,

$$
\left|\frac{\tilde{\Theta}_{\mu, \nu_{4}}(t)-\tilde{\Theta}_{\mu, \nu_{3}}(t)}{\nu_{4}-\nu_{3}}\right|>v_{0} .
$$

With (3-30), (3-45), and (3-48) and by choosing $\nu_{3}$ and $\nu_{4}$ sufficiently close to one another, we have

$$
\begin{aligned}
\left.\frac{\partial \mathbf{P}_{0}}{\partial \nu}\right|_{\nu=\nu_{2}}= & \frac{\tilde{\Theta}_{\mu, \nu_{4}}(t)-\tilde{\Theta}_{\mu, \nu_{3}}(t)}{\nu_{4}-\nu_{3}}+\mathcal{O}\left(\left|\mathbf{P}_{-1}\right|(\mu, \bar{\nu})\right) \\
& +\mathcal{O}\left(\nu^{+}-\nu^{-}\right)
\end{aligned}
$$

Therefore, with (3-42), (3-44), (3-49), and (3-50)) we can write

$$
\nu^{+}-\nu^{-}=\mathcal{Z}(\mu)+\mathcal{O}\left(\mathcal{Z}^{2}(\mu)\right)
$$


where

$$
\mathcal{Z}(\mu)=\frac{4\left|\mathbf{P}_{-1}\right|(\mu, \bar{\nu})\left(\nu_{3}-\nu_{4}\right)}{\tilde{\Theta}_{\mu, \nu_{3}}\left(t_{0}\right)-\tilde{\Theta}_{\mu, \nu_{4}}\left(t_{0}\right)}, \quad t_{0} \in \mathbb{I}_{0} .
$$

Thanks to (3-31) we obtain the following estimate for the width of the homoclinic zone:

$$
\nu^{+}-\nu^{-} \approx \mathcal{Z}_{r}(\mu)=\frac{4\left|\mathbf{R}_{-1}(\mu, \bar{\nu})\right|\left(\nu_{3}-\nu_{4}\right)}{\tilde{\Theta}_{\mu, \nu_{3}}\left(t_{0}\right)-\tilde{\Theta}_{\mu, \nu_{4}}\left(t_{0}\right)} .
$$

With $(3-33),(3-51)$, and $(3-52)$ it follows that

$$
\left(\nu^{+}-\nu^{-}\right)-\mathcal{Z}_{r}(\mu)=\mathcal{O}\left(\mathcal{Z}_{r}^{2}(\mu)\right)
$$

This real approach gives a good estimate of the width of the homoclinic zone with the same precision as before in (3-36). Moreover, it requires only the computation of $\mathbf{P}_{-1}(\mu, \bar{\nu})$ and that of $\tilde{\Theta}_{\mu, \nu}(t)$ for two different values of $\nu$. However, we still need to find a value of $\nu=\bar{\nu}$ such that (3-30) holds.

In what follows we present another way to compute the width: In the new approach, $\boldsymbol{\Gamma}_{u}$ does not need to return near $\boldsymbol{\Gamma}_{s}$ as close as in (3-39). In this way, we will be able to compute the splitting determinant with less precision. This alternative approach consists in looking at the splitting function for complex values of $t$.

\subsection{The Complex Approach}

Now we present another way to compute the first harmonic, with less precision than in the real approach, but with less effort.

Recall that formulas (1-5) and (1-6) already give the following estimate:

$$
\nu^{+}-\nu^{-}=\mathcal{O}(K(\mu, \gamma-2)) .
$$

Moreover, with (3-49) and (3-52), (3-55) gives us a rough estimate of $\left|\mathbf{P}_{ \pm 1}\right|$, i.e., we have $\left|\mathbf{P}_{ \pm 1}\right|(\mu, \nu)=$ $\mathcal{O}(K(\mu, \gamma-2))$.

Take $0 \ll \delta<\varrho$ and $\Delta_{0}=K(\mu, \gamma-2) e^{2 \pi \delta}$ such that $K(\mu, \gamma-2) \ll \Delta_{0}$. Assume that we have found a value of $\nu=\nu_{0}$ such that

$$
K(\mu, \gamma-2) \ll \sup _{t \in \mathbb{I}_{0}} \Theta_{\mu, \nu_{0}}(t) \leq \Delta_{0}
$$

Observe that looking for such a value of $\nu=\nu_{0}$ requires less effort than searching for $\bar{\nu}$ where $\sup _{t \in \mathbb{I}_{0}} \Theta_{\mu, \bar{\nu}}=$ $\mathcal{O}(K(\mu, \gamma-2))$. In particular, we need to compute the splitting function with a relative error only of order $\sup _{t \in \mathbb{I}_{0}} \Theta_{\mu, \nu_{0}}(t)$.

With (3-19), there exists $s \in \mathbb{I}_{0}$ such that

$$
\begin{aligned}
\sup _{t \in \mathbb{I}_{0}} \Theta_{\mu, \nu_{0}}(t)= & \mathbf{P}_{0}\left(\mu, \nu_{0}\right)+\mathbf{P}_{-1}\left(\mu, \nu_{0}\right) e^{-2 i \pi s} \\
& +\mathbf{P}_{1}\left(\mu, \nu_{0}\right) e^{2 i \pi s}+\mathcal{O}_{2}(s) .
\end{aligned}
$$

Since $\left|\mathbf{P}_{ \pm 1}\right|\left(\mu, \nu_{0}\right)=\mathcal{O}(K(\mu, \gamma-2))$, we then conclude that $\mathbf{P}_{0}\left(\mu, \nu_{0}\right)=\mathcal{O}\left(\Delta_{0}\right)$. Since $\Delta_{0} \gg K(\mu, \gamma-2)$, we are not able to compute precisely the first harmonic $\mathbf{P}_{-1}\left(\mu, \nu_{0}\right)$ with the real approach. However, instead of considering $t \in \mathbb{I}_{0}$ as real, we now consider $t$ in the complex interval $[\delta i, \delta i+1]$. Recall that the Fourier coefficients of $\Theta_{\mu, \nu_{0}}(t)$ are

$$
\begin{aligned}
\mathbf{P}_{0}\left(\mu, \nu_{0}\right) & =\int_{0}^{1} \Theta_{\mu, \nu_{0}}(t) d t, \mathbf{P}_{-1}\left(\mu, \nu_{0}\right) \\
& =\int_{0}^{1} e^{2 \pi i t} \Theta_{\mu, \nu_{0}}(t) d t .
\end{aligned}
$$

Since $\Theta_{\mu, \nu_{0}}$ is periodic and analytic in $\mathrm{B}$ defined in (3-16), we have

$$
\mathbf{P}_{-1}\left(\mu, \nu_{0}\right)=\int_{i \delta}^{i \delta+1} e^{2 \pi i t} \Theta_{\mu, \nu_{0}}(t) d t .
$$

With (3-19) we have

$$
\begin{aligned}
e^{2 \pi i t} \Theta_{\mu, \nu_{0}}(t)= & \mathbf{P}_{-1}\left(\mu, \nu_{0}\right)+e^{2 \pi i t} \mathbf{P}_{0}\left(\mu, \nu_{0}\right) \\
& +e^{4 \pi i t} \mathbf{P}_{1}\left(\mu, \nu_{0}\right)+e^{2 \pi i t} \mathcal{O}_{2}(t),
\end{aligned}
$$

where

$$
\mathcal{O}_{2}(t)=\mathcal{O}\left(\sup _{t^{\prime} \in \mathbb{I}_{0}}\left|\Theta_{\mu, \nu_{0}}^{2}\left(i \delta+t^{\prime}\right)\right|\right)
$$

With (3-18) we have

$$
\mathbf{P}_{ \pm 1}\left(\mu, \nu_{0}\right)=\mathcal{O}\left(e^{-2 \pi \varrho}\right) .
$$

Therefore, since $\mathbf{P}_{0}\left(\mu, \nu_{0}\right)=\mathcal{O}\left(\Delta_{0}\right)$, with (3-19) and (3-59), we have

$$
\sup _{t^{\prime} \in \mathbb{I}_{0}}\left|\Theta_{\mu, \nu_{0}}\right|\left(i \delta+t^{\prime}\right)=\mathcal{O}\left(e^{2 \pi \delta-2 \pi \varrho}\right)=\mathcal{O}\left(\left|\mathbf{P}_{-1}\right| e^{2 \pi \delta}\right),
$$

and further we have

$$
\begin{aligned}
\mathbf{P}_{1}\left(\mu, \nu_{0}\right) e^{4 i \pi t} & =\mathcal{O}\left(e^{-2 \pi(\varrho+2 \delta)}\right) \\
\left|e^{2 \pi i t} \mathcal{O}_{2}(t)\right| & =\mathcal{O}\left(\left.\left|e^{+2 \pi i t}\right| \mathbf{P}_{-1}\right|^{2}\left(\mu, \nu_{0}\right) e^{4 \pi \delta} \mid\right) \\
& =\mathcal{O}\left(e^{-2 \pi(2 \varrho-\delta)}\right)
\end{aligned}
$$

We distinguish two cases:

Case 1: $\delta>\frac{\varrho}{3}$. In this case, $2 \varrho-\delta<\varrho+2 \delta$, and from (3-61) we have

$$
\left|\mathbf{P}_{1}\left(\mu, \nu_{0}\right) e^{4 i \pi t}\right| \ll\left|e^{2 \pi i t} \mathcal{O}_{2}(t)\right| .
$$

Using (3-23), we write

$$
\begin{aligned}
e^{2 \pi i t} \Theta_{\mu, \nu_{0}}(t) & =e^{2 \pi i t} \tilde{\Theta}_{\mu, \nu_{0}}(t)+e^{2 \pi i t} \tilde{h}_{\mu, \nu_{0}}(t) \\
& =\mathcal{A}(t)+\mathcal{E}_{1}(t)
\end{aligned}
$$


where $\mathcal{A}(t)=\mathbf{P}_{-1}\left(\mu, \nu_{0}\right)+e^{2 \pi i t} \mathbf{P}_{0}\left(\mu, \nu_{0}\right)$, and with $(3-61)$,

$$
\mathcal{E}_{1}(t)=\mathcal{O}\left(e^{-2 \pi(2 \varrho-\delta)}\right) .
$$

Observe that for $t \in[i \delta, i \delta+1]$, we have

$$
\left|e^{2 i \pi t} \tilde{h}_{\mu, \nu_{0}}(t)\right|=\mathcal{O}\left(e^{-2 \pi(2 \varrho-\delta)}\right) .
$$

In this case

$$
\int_{i \delta}^{i \delta+1} \mathcal{A}(t) d t=\frac{1}{2}(\mathcal{A}(i \delta)+\mathcal{A}(i \delta+1 / 2)) .
$$

But with (3-62), we have

$$
\int_{i \delta}^{i \delta+1} e^{2 \pi i t} \tilde{\Theta}_{\mu, \nu_{0}}(t) d t=\int_{i \delta}^{i \delta+1} \mathcal{A}(t) d t+\mathcal{O}\left(e^{-2 \pi(2 \varrho-\delta)}\right) .
$$

Finally, from (3-57), (3-62), (3-63), and (3-64) we get

$$
\mathbf{P}_{-1}\left(\mu, \nu_{0}\right)=\mathbf{C}_{-1}\left(\mu, \nu_{0}\right)+\tilde{r}_{1}
$$

where

$$
\begin{aligned}
\mathbf{C}_{-1}\left(\mu, \nu_{0}\right) & =\frac{1}{2} e^{-2 \pi \delta}\left(\tilde{\Theta}_{\mu, \nu_{0}}(i \delta)-\tilde{\Theta}_{\mu, \nu_{0}}\left(i \delta+\frac{1}{2}\right)\right), \\
\left|\tilde{r}_{1}\right| & =\mathcal{O}\left(e^{-2 \pi(2 \varrho-\delta)}\right) .
\end{aligned}
$$

Case $2: \delta \leq \frac{\varrho}{3}$. In this case, from $(3-61)$ we have

$$
\left|\mathbf{P}_{1}\left(\mu, \nu_{0}\right) e^{4 i \pi t}\right| \geq\left|e^{2 \pi i t} \mathcal{O}_{2}(t)\right|
$$

Therefore we cannot neglect the term $\mathbf{P}_{1} e^{4 \pi i t}$ from the integration in (3-57). Thus we write

$$
\begin{aligned}
e^{2 i \pi t} \tilde{\Theta}_{\mu, \nu_{0}}(t)= & \mathbf{P}_{-1}\left(\mu, \nu_{0}\right)+e^{2 \pi i t} \mathbf{P}_{0}\left(\mu, \nu_{0}\right) \\
& +\mathbf{P}_{1}\left(\mu, \nu_{0}\right) e^{4 \pi i t}+\mathcal{E}(t) \\
= & \tilde{\mathcal{A}}(t)+\mathcal{E}(t),
\end{aligned}
$$

where with (3-61), we have

$$
\begin{aligned}
\tilde{\mathcal{A}}(t) & =\mathbf{P}_{-1}\left(\mu, \nu_{0}\right)+e^{2 \pi i t} \mathbf{P}_{0}\left(\mu, \nu_{0}\right)+\mathbf{P}_{1} e^{4 \pi i t}, \\
\mathcal{E}(t) & =\mathcal{O}\left(e^{-2 \pi(2 \varrho-\delta)}\right) .
\end{aligned}
$$

In this case,

$$
\begin{gathered}
\int_{i \delta}^{i \delta+1} \tilde{\mathcal{A}}(t) d t=\frac{1}{4}\left(\tilde{\mathcal{A}}(i \delta)+\tilde{\mathcal{A}}\left(i \delta+\frac{1}{2}\right)+\tilde{\mathcal{A}}\left(i \delta+\frac{1}{4}\right)\right. \\
\left.+\tilde{\mathcal{A}}\left(i \delta+\frac{3}{4}\right)\right)
\end{gathered}
$$

and we get

$$
\mathbf{P}_{-1}\left(\mu, \nu_{0}\right)=\mathbf{C}_{-1}\left(\mu, \nu_{0}\right)+\tilde{r}_{2},
$$

where

$$
\begin{aligned}
& \mathbf{C}_{-1}\left(\mu, \nu_{0}\right) \\
& =e^{-2 \pi \delta} \frac{1}{4}\left(\hat{\Theta}_{\mu, \nu_{0}}(0)-\hat{\Theta}_{\mu, \nu_{0}}\left(\frac{1}{2}\right)\right. \\
& \left.\quad-i\left(\hat{\Theta}_{\mu, \nu_{0}}\left(\frac{1}{4}\right)-\Theta_{\mu, \nu_{0}}\left(\frac{-1}{4}\right)\right)\right),
\end{aligned}
$$

and

$$
\left|\tilde{r}_{2}\right|=\mathcal{O}\left(e^{-2 \pi(2 \varrho-\delta)}\right)=\mathcal{O}\left(\mathbf{C}_{-1}^{2}\left(\mu, \nu_{0}\right) e^{2 \pi \delta}\right) .
$$

When $\delta>\varrho / 3$, the estimate given in $(3-65)$ requires the computation of the splitting determinant $\tilde{\Theta}_{\mu, \nu_{0}}(t)$ at only two different values of $t$. However, when $\delta \leq \varrho / 3$, (3-66) requires four different values of $t$. The computation in the first case is faster, but since $\delta$ is bigger, we lose some precision.

From the complex approach, the width of the homoclinic zone is approximated by

$$
\nu^{+}(\mu)-\nu^{-}(\mu) \approx \mathcal{Z}_{c}(\mu)
$$

where

$$
\mathcal{Z}_{c}(\mu)=4 \frac{\left|\mathbf{C}_{-1}\left(\mu, \nu_{0}\right)\right|\left(\nu_{3}^{\prime}-\nu_{4}^{\prime}\right)}{\tilde{\Theta}_{\mu, \nu_{3}^{\prime}}(t)-\tilde{\Theta}_{\mu, \nu_{4}^{\prime}}(t)}
$$

with $\nu_{3}^{\prime}$ and $\nu_{4}^{\prime}$ chosen near $\nu_{0}$. Since $\mathbf{C}_{-1}\left(\mu, \nu_{0}\right)=$ $\mathcal{O}\left(e^{-2 \pi \varrho}\right)$ with $(3-65)$ or $(3-66)$, we have

$$
\left|\mathcal{Z}_{c}(\mu)-\left(\nu^{+}(\mu)-\nu^{-}(\mu)\right)\right|=\mathcal{O}\left(\mathbf{C}_{-1}^{2}\left(\mu, \nu^{+}\right) e^{2 \pi \delta}\right) .
$$

\subsection{Real versus Complex}

The real approach provides a good estimate of the width of the homoclinic zone. More precisely, formula (3-53) gives an estimate of the width with a relative error of the same order; see (3-54).

However, this approach requires that one compute the splitting determinant with the same relative error. This task becomes more and more delicate as the main parameter approaches zero.

The complex approach requires less precision for the computation of the splitting determinant (and therefore can be computed much faster) as $\delta$ is chosen larger. However, the estimate of the width is obtained with less precision.

In the case of the Bogdanov map, we use similar notation: $b$ is the slave parameter and $a$ is the main parameter. The first harmonic computed with $(3-31)$ is denoted by $\mathbf{R}_{-1}(a, \bar{b})$, where $\bar{b}$ is the analogue of $\bar{\nu}$ in the case of 



FIGURE 2. Left: (I) Graph of $\log _{10}\left|e^{2 \pi \delta}\right|$ against the parameter $a$. Recall that $\delta$ is chosen such that $K(\mu, \gamma-2) \ll$ $\sup _{t \in \mathbb{I}_{0}} \Theta_{\mu, \nu_{0}}(t) \leq \Delta_{0}=\mathcal{O}\left(\mathbf{P}_{0}\left(\mu, \nu_{0}\right)\right)$ (3-56). (II) Graph of $\log _{10}\left|\mathbf{C}_{1}\left(a, b_{0}\right)\right|$ against the parameter $a$, which essentially coincides with the graph of $\log _{10}\left|\mathbf{R}_{1}(a, \bar{b})\right|$ against $a$. (III) The corresponding error, i.e., $\log _{10}\left|\mathbf{C}_{1}\left(a, b_{0}\right)-\mathbf{R}_{1}(a, \bar{b})\right|$ against a. Right: (IV) Computation of the magnitude of the homoclinic zone with the real approach, i.e., $\log _{10}\left(\mathcal{Z}_{r}(a)\right)$ against a. (V) The graph of $\log _{10}\left|\mathcal{Z}_{r}(a)-\mathcal{Z}_{c}(a)\right|$ against $a$.

the quadratic map. Similarly, $\mathbf{C}_{-1}\left(a, b_{0}\right)$ stands for the first harmonic computed with (3-65) or (3-66), where $b_{0}$ is the analogue of $\nu_{0}$ in the case of the quadratic map.

For illustration, we compute the first harmonic and the width of the homoclinic zone using both approaches for the Bogdanov map $(\tilde{\gamma}=3)$; see Figure 2 .

We easily verify that

$$
\begin{aligned}
& \log _{10}\left(\mathbf{C}_{-1}\left(a, b_{0}\right)-\mathbf{R}_{-1}(a, \bar{b})\right) \\
& \quad \approx 2 \log _{10}\left(|\mathbf{R}|_{-1}(a, \bar{b})\right)+\log _{10}\left(e^{2 \pi \delta}\right),
\end{aligned}
$$

which follows from (3-54) and (3-68). Furthermore, we also verify that

$$
\begin{aligned}
\log _{10} & \left|\mathcal{Z}_{r}(a)-\mathcal{Z}_{c}(a)\right| \\
& \approx \log _{10}\left(\frac{\left|\mathbf{C}_{-1}\left(a, b_{0}\right)\right| e^{2 \pi \delta}\left(b_{3}^{\prime}-b_{4}^{\prime}\right)}{\tilde{\Theta}_{a, b_{3}^{\prime}}\left(t_{0}\right)-\tilde{\Theta}_{a, b_{4}^{\prime}}\left(t_{0}\right)}\right) \\
& \approx \log _{10}\left(b^{+}-b^{-}\right)+\log _{10}\left|\mathbf{C}_{-1}\left(a, b_{0}\right) e^{2 \pi \delta}\right|,
\end{aligned}
$$

where $b_{3}^{\prime}, b_{4}^{\prime}$ are the analogues of $\nu_{3}^{\prime}, \nu_{4}^{\prime}$.

Example 3.4. We consider the Bogdanov map with $a \approx 7 \times 10^{-5}$. Using the real approach, we have $\log _{10}\left(b^{+}-b^{-}\right) \approx-1000$; see Figure 2. With this approach, we compute $\tilde{\Theta}_{a, \bar{b}}$ with a relative error of order $10^{-1000}$, which is already a quite delicate task. However, from the complex approach, we can (for instance) choose $\delta$ in such a way that $e^{2 \pi \delta} \approx 10^{700}$; see again Figure 2 .

In this way, for values of $t \in[i \delta, i \delta+1]$, we have

$$
\log _{10}\left(\tilde{\Theta}_{a, b_{0}}(t)\right) \approx \log \left(\mathbf{C}_{1}\left(a, b_{0}\right) e^{2 \pi \delta}\right) \approx-300 .
$$

Therefore, computing $\mathbf{C}_{1}\left(a, b_{0}\right)$ with (3-66) requires the computation of the splitting determinant with a rel- ative error or order $10^{-300}$. Moreover, we just need to find a first value of $b=b_{0}$ such that

$$
\log _{10} \sup _{t \in \mathbb{I}_{0}}\left\|\boldsymbol{\Gamma}_{u}(t)-\boldsymbol{\Gamma}_{s}(t)\right\| \approx-300 .
$$

However, instead of having a relative error for the width of order $10^{-1000}$ as with the real approach, we obtain an estimate of the width with a relative error of order $10^{-300}$.

Now that we can compute the width of the homoclinic zone, we do so for $\tilde{n}$ (several hundred) values of $\mu^{1 / 4}$ and establish the set

$$
\begin{gathered}
\mathcal{H}=\left\{\left(\mu_{i}^{\frac{1}{4}}, \log \left(\delta_{i}\right)\right), \delta_{i}=\nu^{+}\left(\mu_{i}\right)-\nu^{-}\left(\mu_{i}\right), c<\mu_{i}<d,\right. \\
i=1, \ldots, \tilde{n}\} .
\end{gathered}
$$

In what follows, we describe how we extract the corresponding coefficients from the ansatz (1-8).

\subsection{Extracting the Coefficients}

Recall that the ansatz we shall consider takes the form $(2-3)$, where the $f_{n}$ 's satisfy $(2-2)$. From the set $\mathcal{H}$ defined by (3-69) we construct the following matrices:

$$
\mathbf{A}=\left(A_{i, j}\right)_{i=0, \ldots, \tilde{n}-1, j=1, \ldots, \tilde{n}}, \quad A_{i, j}=f_{i}\left(\mu_{j}^{1 / 4}\right) .
$$

In the case of the Bogdanov map, the set of normalized data is defined in (3-2), that is, the $\mu_{i}^{1 / 4}$, s above are replaced by $a^{1 / 2}$.

Let

$$
\alpha=\left(\alpha_{1}, \ldots, \alpha_{\tilde{n}}\right)=\mathbf{A}^{-1} \cdot \mathbf{w},
$$

where $\mathbf{w}=\left(\log \delta_{1}, \ldots, \log \delta_{\tilde{n}}\right)$. Observe that

$$
\sum_{i=0}^{\tilde{n}-1} \alpha_{i} f_{i}\left(\mu_{j}^{1 / 4}\right)=\log \delta_{j}, \quad \forall j=1, \ldots, \tilde{n},
$$




\begin{tabular}{|c|c|c|}
\hline Coeff. & Scale & Hénon Map \\
\hline$\tilde{A}_{0}$ & 1 & $2.4744255935532510538408 \times 10^{6}$ \\
$\tilde{A}_{1}$ & $|\tilde{a}-1|^{1 / 4}$ & $-2.878113364919828141704 \times 10^{6}$ \\
$\tilde{A}_{2}$ & $|\tilde{a}-1|^{1 / 2}$ & $1.8211174314566012763528 \times 10^{6}$ \\
$\tilde{A}_{3}$ & $|\tilde{a}-1|^{3 / 4}$ & -412552.07921345800366019 \\
$\tilde{A}_{4}$ & $|\tilde{a}-1|$ & -309961.28583121907079391 \\
$\tilde{A}_{5}$ & $|\tilde{a}-1|^{5 / 4}$ & 257055.93487794037812901 \\
$\tilde{A}_{6}$ & $|\tilde{a}-1|^{3 / 2}$ & -56830.201956139947433580 \\
$\tilde{A}_{7}$ & $|\tilde{a}-1|^{7 / 4}$ & -12386.990577003086404843 \\
$\tilde{A}_{8}$ & $|\tilde{a}-1|^{2}$ & -11792.964908478734939516 \\
$\tilde{A}_{9}$ & $|\tilde{a}-1|^{9 / 4}$ & 18742.189161591275288347 \\
$\tilde{A}_{10}$ & $|\tilde{a}-1|^{5 / 2}$ & -4774.6727458595190485600 \\
$\tilde{A}_{11}$ & $|\tilde{a}-1|^{11 / 4}$ & -2822.9663193640187675835 \\
$\tilde{A}_{12}$ & $|\tilde{a}-1|^{3}$ & 3276.6438736125169964394 \\
$\tilde{A}_{13}$ & $|\tilde{a}-1|^{13 / 4}$ & -1910.5466958542171966392 \\
$\tilde{A}_{14}$ & $|\tilde{a}-1|^{7 / 2}$ & 7704.6605615546853854041 \\
$\tilde{A}_{15}$ & $|\tilde{a}-1|^{15 / 4}$ & -7827.0351891507566506398 \\
$\tilde{A}_{16}$ & $|\tilde{a}-1|^{4}$ & 13919.102717097324631620 \\
$\tilde{A}_{17}$ & $|\tilde{a}-1|^{17 / 4}$ & -11932.139780641352182621 \\
$\tilde{A}_{18}$ & $|\tilde{a}-1|^{9 / 2}$ & 22120.721696311178434645 \\
\hline
\end{tabular}

TABLE 2. The 19 first coefficients in $(2-6)$. All the given digits are correct. We also conjecture that the series (2-6) belongs to the Gevrey-1 class.

that is, the coefficients $\alpha_{i}$ have been constructed in such a way that the map

$$
\begin{aligned}
\phi^{\{\tilde{n}\}}:\left(0, \varepsilon_{0}\right) & \rightarrow \mathbb{R}, \\
x & \mapsto \phi^{\{\tilde{n}\}}(x)=\sum_{i=0}^{\tilde{n}} \alpha_{i} f_{i}(x)
\end{aligned}
$$

interpolates the set of data $\mathcal{H}$.

To illustrate our techniques, Table 1 indicates the first coefficients of the interpolation $(\tilde{n} \approx 100)$ in the case of the Bogdanov map (left, $\tilde{\gamma}=3$ ) and in the case of the quadratic map (right, $\gamma=-3$ ). In the case of the Hénon map, replacing the ansatz $(1-8)$ by $(2-7)$, we obtain the coefficients indicated in Table 2.

Redoing the above interpolation for different values of $\gamma$ reveals that the first nonlinear term in the expansion satisfies

$$
N_{1}(\gamma)=-\left(\frac{6(\gamma-2)}{7 \sqrt{2}}\right)^{2}
$$

in the case of the quadratic map, and

$$
B_{1}(\tilde{\gamma})=-\left(\frac{6 \tilde{\gamma}}{7}\right)^{2}
$$

in the case of the Bogdanov map. These equalities have been verified to high precision. More precisely, we show that (3-71) and (3-72) are verified up to the same number of correct digits as in (4-7) when we check the extrapolation to zero; see Section 4.3 below for more details.

\section{VALIDATION OF THE NUMERICAL METHOD}

To test the validity of our result, we propose three tests. First, we test the validity of the ansatz. In what follows, the experiments are presented in the cases of the Bogdanov map and the Hénon map, but the same test can be applied in the case of the quadratic map, thereby confirming formula $(2-5)$.

\subsection{Extrapolability}

We claim that the ansatz $(1-8)$ is appropriate for an asymptotic expansion of the width if the following criterion is satisfied.

Assume that a function $G:\left(0, \varepsilon_{0}\right) \rightarrow \mathbb{R}$ possesses the following asymptotics at 0 :

$$
G(x) \asymp \sum_{i=0}^{\infty} \alpha_{i} f_{i}(x),
$$

where $\left\{f_{i}(x), i \in \mathbb{N}\right\}$ is the asymptotic sequence defined in $(2-2)$.

Define

$$
G^{\{3 k+3\}}(x)=\sum_{i=0}^{3 k+3} \alpha_{i} f_{i}(x)
$$

We have

$$
\left|G(x)-G^{\{3 k+3\}}(x)\right|=x^{2 k+3}\left(\alpha_{3 k+4}+\varepsilon_{1}(x)\right),
$$

where $\varepsilon_{1}(x)=\mathcal{O}(x)$. From $(4-2)$ we get

$$
\begin{aligned}
& \log \left|G(x)-G^{\{3 k+3\}}(x)\right| \\
& \quad=\log \left|\alpha_{3 k+4}\right|+(2 k+3) \log x+\log (1+\varepsilon(x)) \\
& \quad=\log \left|\alpha_{3 k+4}\right|+(2 k+3) \log x+\varepsilon_{2}(x),
\end{aligned}
$$

where $\varepsilon_{2}(x)=\mathcal{O}(|x|)$.

This implies that the quantity $\log \left|G(x)-G^{\{3 k+3\}}(x)\right|$ is approximatively linear in $\log x$. This must be satisfied for values of $x$ outside the data set used for interpolation.

Now we apply this criterion to the Bogdanov family. Recall that $a$ is the slave parameter and $b$ is the main parameter. Take an interval $\left[c^{\prime}, d^{\prime}\right]$, where $c<c^{\prime}<d^{\prime}<$ $d$, and consider the interpolation of the set $\tilde{\mathcal{H}}$ for values of $a$ in $\left[c^{\prime}, d^{\prime}\right]$. In other words, we consider the set

$$
\tilde{\mathcal{H}}^{\prime}=\left\{\left(a^{1 / 2}, \log \delta(a)\right) \in \tilde{\mathcal{H}} \mid c^{\prime}<a<d^{\prime}\right\}
$$

which consists of $3 k+4$ different values, and construct the corresponding set of coefficients $\left\{\alpha_{i}\right\}_{i=0, \ldots, 3 k+3}$ as described in Section 3. 




FIGURE 3. Plot of the set $\mathcal{L}_{c, d}(4-4)$ for the Bogdanov map in the case $\tilde{\gamma}=3, c=3.5 \times 10^{-5}, d=9.4 \times 10^{-3}$, $\tilde{n}=140, k=36$.

We plot the set

$$
\begin{gathered}
\mathcal{L}_{c, d}=\left\{\left(\log (a), \log \left|G^{\{3 k+3\}}(\sqrt{a})-\left(b^{+}(a)-b^{-}(a)\right)\right|\right),\right. \\
c<a<d\}
\end{gathered}
$$

in Figure 3: $\tilde{n}=140, k=36, c=3.5 \times 10^{-5}, d=$ $9.4 \times 10^{-3}$. The bold line shows the interval $\left[c^{\prime}, d^{\prime}\right]$. From $(4-1)$ and $(4-2)$ we must get

$$
\begin{aligned}
\log \left|G^{\{3 k+3\}}(\sqrt{a})-\left(b^{+}(a)-b^{-}(a)\right)\right| & \approx 37 \log a+\mathrm{C} \\
& =74 \log \sqrt{a}+\mathrm{C},
\end{aligned}
$$

where $\mathrm{C}$ is a constant. In Figure 3, the set (4-4) looks like a straight line with slope approximately 75 , which indicates that the ansatz (1-8) satisfies the above criterion.

In the Hénon case, we interpolate the set of data (3-3) with the polynomial ansatz (2-1), and the normalized width takes the form

$$
\frac{\tilde{b}^{+}(\tilde{a})-\tilde{b}^{-}(\tilde{a})}{K(1-\tilde{a}, 0)} \asymp \sum_{i=0}^{\tilde{n}} \tilde{A}_{i}(1-\tilde{a})^{i / 4}
$$

We test the polynomial expansion in the same way that we test the Dulac expansion for the Bogdanov case. More precisely, writing

$$
\tilde{G}^{\{\tilde{k}-1\}}(x)=\sum_{i=0}^{\tilde{k}-1} \tilde{A}_{i} x^{i}, \quad \tilde{G}(x) \asymp \sum_{i=0}^{\infty} \tilde{A}_{i} x^{i},
$$

we have

$$
\log \left|\tilde{G}(x)-\tilde{G}^{\{\tilde{k}-1\}}(x)\right|=\log \left|\tilde{A}_{\tilde{k}}\right|+\tilde{k} \log x+\mathcal{O}(x),
$$

and replacing $x$ by $(\tilde{a}-1)^{1 / 4}$ in $(4-5)$ leads to

$$
\begin{aligned}
\log \mid \tilde{G} & \left((\tilde{a}-1)^{1 / 4}\right)-\tilde{G}^{\{\tilde{k}\}}\left((\tilde{a}-1)^{1 / 4}\right) \mid \\
& =\log \left|\tilde{A}_{\tilde{k}}\right|+\frac{\tilde{k}}{4} \log (\tilde{a}-1)+\mathcal{O}\left((\tilde{a}-1)^{1 / 4}\right) .
\end{aligned}
$$



FIGURE 4. Plot of the set $\tilde{\mathcal{L}}_{c, d}(4-6)$ for the Hénon map, $c=1.69 \times 10^{-10}, d=1.125 \times 10^{-7}, \tilde{k}=60$, $\tilde{n}=140$.

The set

$$
\begin{aligned}
\tilde{\mathcal{L}}_{c, d}=\{( & \log (\tilde{a}-1), \\
& \left.\log \left|\tilde{G}^{\{\tilde{k}\}}\left((\tilde{a}-1)^{1 / 4}\right)-\left(\tilde{b}^{+}(\tilde{a})-\tilde{b}^{-}(\tilde{a})\right)\right|\right), \\
& c<\tilde{a}-1<d\}
\end{aligned}
$$

is plotted (with $\tilde{k}=60, c=1.69 \times 10^{-10}, d=1.125 \times$ $10^{-7}$ ) in Figure 4. The graph mimics a straight line of slope $\approx 15=60 / 4$, meaning that the polynomial ansatz satisfies the above criteria.

The second experiment consists in checking the stability of our interpolation as we (randomly) change the data $\tilde{\mathcal{H}}$.

\subsection{Checking Numerical Stability}

In this section, our interest is with the precision of our data for the normalized width of the homoclinic zone that is required to produce reliable results for the coefficients. The result of our test is presented in the case of the Bogdanov map. That is, we test the asymptotic expansion (2-6).

In order to simulate round-off errors, we modify the data in the $N$ th digit by adding a random perturbation of order $10^{-N}$ to every value of the normalized width and recompute the coefficients of the asymptotic expansion using the procedure described in Section 3. We repeat the experiment for several values of $N$.

Figure 5 concerns the coefficients $A_{11}$ in $(2-6)$ : for each value of $N$, we recompute the corresponding coefficient (denoted by $A_{11}^{\{N\}}$ ) after adding a random perturbation of order $10^{-N}$. The figure clearly indicates that the precision of the computation decreases linearly with respect to $N$, and if the number of correct digits in the data is less than $N=170$, the corresponding coefficient cannot be computed correctly. However, if $N=200$, 


\begin{tabular}{|c|c|c|}
\hline$\tilde{\gamma}$ & $\Theta(\tilde{\gamma})$ & $-\log _{10}\left|\left(\Theta(\tilde{\gamma})-\exp \left(A_{\mathbf{0}}(\tilde{\gamma})\right)\right) / \Theta(\tilde{\gamma})\right|$ \\
\hline-2 & 0.28524883190581352 & 65.23 \\
0 & $2.47442559355325105 \times 10^{6}$ & 90.01 \\
3 & $4.05522622851113044 \times 10^{26}$ & 62.04 \\
6 & $2.70980378082897208 \times 10^{47}$ & 60.03 \\
7 & $3.09943158275750458 \times 10^{54}$ & 59.6 \\
9 & $5.18377311752952789 \times 10^{68}$ & 55,6 \\
\hline
\end{tabular}

TABLE 3. The value of $\boldsymbol{\Theta}(\tilde{\gamma})$ for different values of $\tilde{\gamma}$. We clearly observe that the splitting constant coincides with the first term in $(2-6)$ up to at leat the first 50 digits.

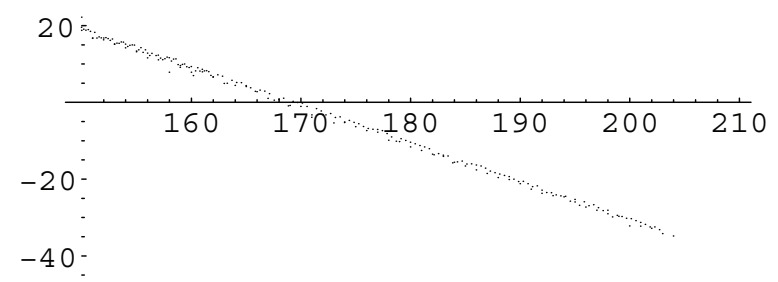

FIGURE 5. The relative error $\log _{10}\left|\frac{A_{11}-A_{11}^{\{N\}}}{A_{11}}\right|$ plotted against $N$ for the Bogdanov map with $\tilde{\gamma}=3$.

the corresponding coefficient is computed with 30 correct digits.

\subsection{Extrapolation to Zero}

As announced in Section 2, for each family we are able to define the splitting constant associated with the "unperturbed map"; see [Gelfreich and Naudot 06] for more details.

In what follows, our discussion concerns the Bogdanov family. The splitting constant is denoted by $\boldsymbol{\Theta}(\tilde{\gamma})$. Using formula (2-6), we have

$$
\exp \left(A_{0}(\tilde{\gamma})\right)=\boldsymbol{\Theta}(\tilde{\gamma})
$$

Since we can independently compute the invariant $\boldsymbol{\Theta}(\tilde{\gamma})$ with very high precision, we can easily check the validity of our computation for the first term of the asymptotic expansion.

Table 3 indicates, for different values of $\tilde{\gamma}$, the values of $\boldsymbol{\Theta}(\tilde{\gamma})$ (left) computed with 20 correct digits. For each value of $\tilde{\gamma}$, we observe that $(4-7)$ holds, and we indicate the relative error represented by $-\log _{10} \mid(\Theta(\tilde{\gamma})-$ $\left.\exp \left(A_{0}(\tilde{\gamma})\right)\right) / \boldsymbol{\Theta}(\tilde{\gamma}) \mid$ in the right-hand column.

\section{ACKNOWLEDGMENTS}

This work is supported by EPSRC grant EP/COOO595/1.

\section{REFERENCES}

[Arnol'd 72] V. I. Arnol'd. "Lecture on Bifurcations in Versal Systems." Russ Math. Surveys 27 (1972), 54-123.

[Arnol'd 83] V. I. Arnol'd. Geometrical Methods in the Theory of Ordinary Differential Equations. New York: Springer, 1983.

[Arrowsmith 93] D. K. Arrowsmith. "The Bogdanov Map: Bifurcation, Mode Locking, and Chaos in a Dissipative System." Int. J. Bifurcation and Chaos 3 (1993), 803-842.

[Arrowsmith and Place 90] D. K. Arrowsmith and C. M. Place. An Introduction to Dynamical Systems. Cambridge: Cambridge University Press, 1990.

[Bazykin et al. 89] A. D. Bazykin, Y. A. Kuznetsov, and A. I. Khibnik. Portraits of Bifurcations: Bifurcation Diagrams of Planar Dynamical Systems. Moscow: Znanie, 1989.

[Bogdanov 75] R. I. Bogdanov. "Versal Deformations of a Singular Point on the Plane in the Case of Zero Eigenvalues." Functional Analysis and Its Applications 9:2 (1975), 144-145.

[Bogdanov 81] R. I. Bogdanov. "Bifurcation of the Limit Cycle of a Family of Plane Vector Fields." Trudy Sem. Petrovsk. 2 (1976), 23-35. English translation in Sel. Math. Sov. 1:4 (1981), 373-387.

[Brännström and Gelfreich 08] N. Brännström and V. Gelfreich. "Asymptotic Series for the Splitting of Separatrices near a Hamiltonian Bifurcation." arXiv:0806.2403v1 [math.DS], 2008.

[Broer and Roussarie 01] H. W. Broer and R. Roussarie. "Exponential Confinement of Chaos in the Bifurcation Sets of Real Analytic Diffeomorphisms." In Global Analysis of Dynamical Systems, pp. 167-210. Bristol: Inst. Phys., 2001.

[Broer et al. 91] H. W. Broer, F. Dumortier, S. J. Van Strien, and F. Takens. Structures in Dynamics, Finite Dimensional Deterministic Studies, Studies in Mathematical Physics 2. Amsterdam: North-Holland, 1991.

[Broer et al. 92] H. W. Broer, R. Roussarie, and C. Simó. "A Numerical Survey on the Takens-Bogdanov Bifurcation for Diffeomorphisms." In European Conference on Iteration Theory, 89, edited by C. Mira et al., pp. 320-334. Singapore: World Scientific, 1992. 
[Broer et al. 96] H. W. Broer, R. Roussarie, and C. Simó "Invariant Circles in the Bogdanov-Takens Bifurcation for Diffeomorphisms." Ergodic Th. Dynam. Syst. 16:6 (1996), $1147-1172$.

[Delshams and Ramirez-Ros 99] A. Delshams and R. Ramirez-Ros. "Singular Separatrix Splitting and the Melnikov Method: An Experimental Study." Experiment. Math. 8:1 (1999), 29-48.

[Dumortier et al. 81] F. Dumortier, P. R. Rodrigues, and R. Roussarie. Germs of Diffeomorphisms in the Plane, Lecture Notes in Mathematics 902. New York: Springer, 1981.

[Fontich and Simó 90] E. Fontich and C. Simó. "The Splitting of Separatrices for Analytic Diffeomorphisms." Ergodic Theory Dynam. Syst. 10:2 (1990), 295-318.

[Gelfreich 96] V. G. Gelfreich. "Conjugation to a Shift and Splitting of Separatrices." Applicationes Mathematicae 24:2 (1996), 127-140.

[Gelfreich 99] V. G. Gelfreich. "A Proof of the Exponentially Small Transversality of the Separatrices for the Standard Map." Comm. Math. Phys. 201 (1999), 155-216.

[Gelfreich 00] V. G. Gelfreich. "Splitting of a Small Separatrix Loop near the Saddle-Center Bifurcation in AreaPreserving Maps." Physica D 136 (2000), 266-279.

[Gelfreich 01] V. G. Gelfreich and D. Sauzin. "Borel Summation and the Splitting of Separatrices for the Hénon Map." Annales l'Institut Fourier 51:2 (2001), 513-567.

[Gelfreich 03] V. G. Gelfreich. "Chaotic Zone in the Bogdanov-Takens Bifurcation for Diffeomorphisms." In Analysis and Applications: ISAAC 2001, edited by H. Begehr, R. Gilbert, and Man-Wah Wong, pp. 187-197. Dordrecht: Kluwer, 2003.

[Gelfreich and Naudot 06] V. G. Gelfreich and V. Naudot. "Analytic Invariants Associated with a Parabolic Fixed Point in $\mathbb{C}^{2}$." Preprint, 2006.

[Gelfreich and Simó 09] V. Gelfreich and C. Simó. "HighPrecision Computations of Divergent Asymptotic Series and Homoclinic Phenomena." To appear in Discrete and Continuous Dynamical Systems, 2009.

[Hénon 76] M. Hénon. "A Two-Dimensional Mapping with a Strange Attractor." Commun. Math. Phys. 50 (1976), 69-78.
[Kirchgraber and Stoffer 06] U. Kirchgraber and D. Stoffer. "Transversal Homoclinic Points of the Hńon Map." Annali di Matematica 185 (2006), 187-204.

[Lazutkin 91] V. F. Lazutkin. "An Analytic Integral along the Separatrix of the Semistandard Map: Existence and an Exponential Estimate for the Distance between the Stable and Unstable Separatrices." Algebra i Analiz 4:4 (1992), 110-142 (in Russian); translation in St. Petersburg Math. J. 4:4 (1993), 721-748.

[Lazutkin 05] V. F. Lazutkin. "Splitting of Separatrices for the Chirikov Standard Map." VINITI 6372:84 (1984) (in Russian); translation in J. Math. Sci. (N.Y.) 128:2 (2005), $2687-2705$.

[Levallois and Tabanov 93] P. Levallois and M. B. Tabanov. "Séparation des séparatrices du billard elliptique pour une perturbation algébrique et symétrique de l'ellipse." $C . R$. Acad. Sci. Paris Sr. I Math. 316:6 (1993), 589-592.

[Marděsic 94] P. Marděsic. Chebyshev Systems and the Versal Unfolding of the Cusp of Order n, Travaux en cours 57. Paris: Hermann, 1998.

[Neishtadt 84] A. I. Neishtadt. "The Separation of Motions in Systems with Rapidly Rotating Phase." J. Appl. Math. Mech. 48 (1984), 133-139.

[Ramirez-Ros 05] R. Ramirez-Ros. "Exponentially Small Separatrix Splittings and Almost Invisible Homoclinic Bifurcations in Some Billiard Tables." Phys. D, 210 (2005), 149-179.

[Simó 00] C. Simó. "Analytical and Numerical Detection of Exponentially Small Phenomena." In International Conference on Differential Equations (Proceedings Equadiff '99, Berlin, Germany, August 1-7, 1999), vol. 2, edited by B. Fiedler et al., pp. 967-976. Singapore: World Scientific, 2000 .

[Tabanov 94] M. B. Tabanov. "Separatrices Splitting for Birkhoff's Billiard in Symmetric Convex Comain, Closed to an Ellipse." Chaos, 4:4 (1994), 595-606.

[Palis and Takens 93] J. Palis and F. Takens. Hyperbolicity and Sensitive Chaotic Dynamics at Homoclinic Bifurcations. Fractal Dimensions and Infinitely Many Attractors. Cambridge: Cambridge University Press, 1993.

[Takens 74] F. Takens. "Forced Oscillations and Bifurcations. Applications of Global Analysis, I." In Sympos. Utrecht State Univ., Utrecht, 1973, pp. 1-59, Comm. Math. Inst. Rijksuniv. Utrecht. Utrecht: Rijksuniv., 1974.

V. Gelfreich, Mathematics Institute, University of Warwick, Coventry CV4 7AL, UK (V.Gelfreich@warwick.ac.uk)

V. Naudot, Mathematics Institute, University of Warwick, Coventry CV4 7AL, UK (vnaudot@fau.edu)

Received June 28, 2008; accepted November 18, 2008. 